\title{
OFFSET MASKING IN A DIVIDED VISUAL FIELD STUDY
}

\section{BY}

\section{Keith Young}

Submitted to the graduate degree program in Cognitive Psychology and the Faculty of the Graduate School of the University of Kansas

In partial fulfillment of the requirements for the degree of

Master's of Arts

\author{
Chairperson
}

Committee members

Date defended: 
The Thesis Committee for Keith Young certifies

That this is the approved version of the following thesis:

\section{OFFSET MASKING IN A DIVIDED VISUAL FIELD STUDY}

\section{Committee:}

Chairperson

Date approved: 


\begin{abstract}
A problem in divided visual field studies which use event-related potentials as a dependent measure is the large number of horizontal eye movements participants make during experimental trials. Past attention research suggests that eye movements to lateralized targets should be significantly reduced using a dynamic, offset mask, causing a reduction in attentional capture. The current study attempted to replicate past divided visual field language studies using offset masking procedures. Using a basic offset procedure, eye movements were not reduced in Experiment 1. Experiment 3, however, did see a significant reduction in eye movements using a dynamic offset masking procedure developed in Experiment 2. Low accuracy rates were a concern throughout. In conclusion, horizontal eye movements can be reduced with a dynamic offset procedure but the low accuracy rates and the inconsistent behavioral findings throughout the study do not support using this technique.
\end{abstract}




\section{Acknowledgements}

I hereby acknowledge all of the following people and groups for their help and support that allowed me to successfully complete this thesis. I would first like to thank the faculty and staff of the Cognitive Program and the Department of Psychology at the University of Kansas for giving me the opportunity to learn and work in a supportive and caring environment. I would like to thank my advisor, Dr. Ruth Ann Atchley, for her unending patience and guidance. She not only helped me develop this project but also continued to offer support and advice (both personal and professional) throughout. My gratitude also goes out to Dr. Paul Atchley for his input on this project and continuing faith in my abilities as a researcher and a person. My thanks also go out to Dr. Greg Simpson for being on my defense committee and for his helpful input and insightful questions. I also want to thank all of the other graduate students in the program who not only offer support and help but create such a great environment both in and outside of work.

I would also like to thank all of my family and friends who have helped me complete this work through their unconditional love and support of me as a professional-in-training and as a person. To Mom and Pops: I can never thank you enough for all that you have done for me so I will simply say I love you and thanks for being truly wonderful parents. To Dad: I hope that I have made you proud and I love you, wherever you are. To Paul W., Rob W., Justin, Paul B., Rob B., and Kathryn (and Nate): I just want you all to know that you are truly wonderful siblings and have made my life so enjoyable and I cannot thank you enough for your love and 
support. Finally, to Nate, Duane, Jimmy, Jordan, Sam, Jeff, Scott, Lindsey, Alison, and Aminda: thank you from the bottom of my heart. I could not ask for a better group of friends. 
Table of Contents

Section

Page

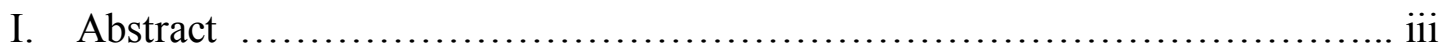

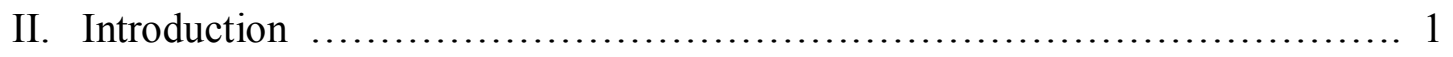

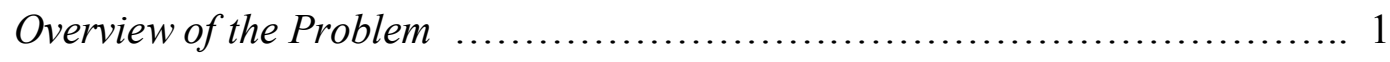

Stimulus-driven and Goal-driven Attentional Capture ....................... 6

Eye Movements and Attentional Capture ................................ 13

Possible Solution to the Problem ........................................ 16

III. Experiment 1 ................................................ 17

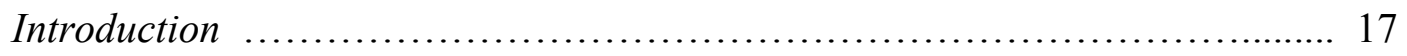

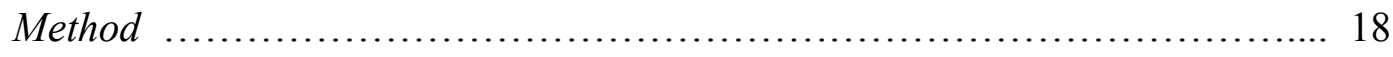

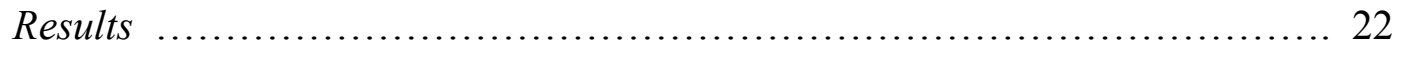

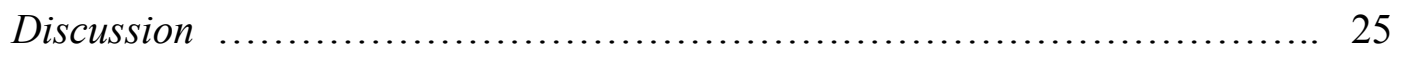

IV. Experiment 2 .................................................. 27

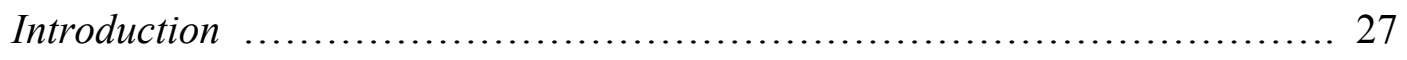

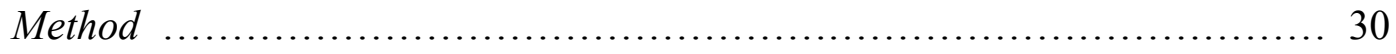

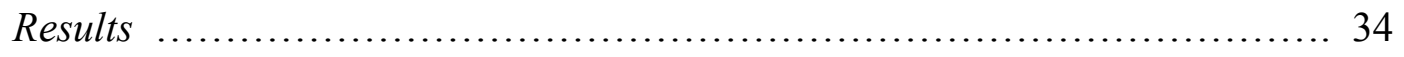

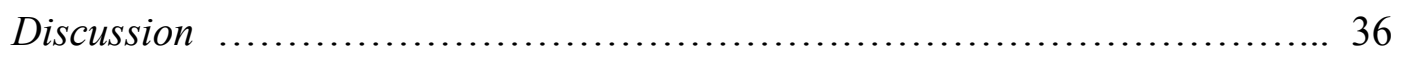

V. Experiment 3 ................................................. 39

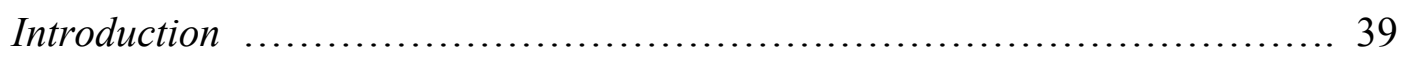

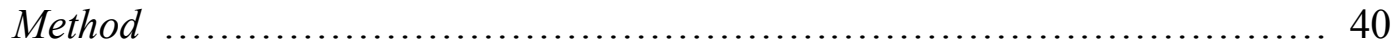

Results ........................................................ 43 


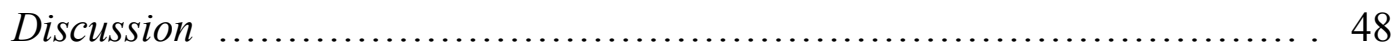

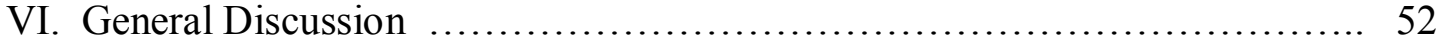

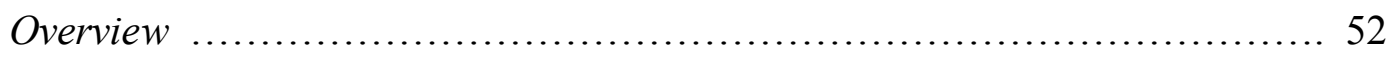

Eye Movements ................................................. 53

Semantic Relatedness Main Effect in Experiment 3 ....................... 57

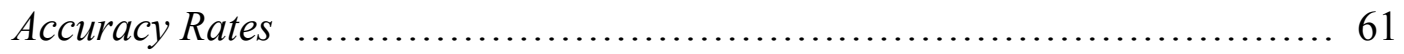

Implications for DVF Methodology .................................... 64

Future Directions .................................................. 65

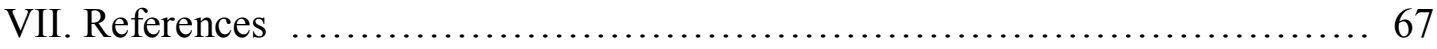


Introduction

\section{Overview of the Problem}

A common concern among researchers who study psychology using eventrelated potentials (ERPs) is the loss of data due to eye movements made by the participants. When participants blink their eyes or shift them left to right (horizontal saccades) a large, unwanted electric field is generated and propagates from the front to the back of the scalp. This electric field generated by the eyes (electrooculargram, or EOG) is measured by electrodes that are typically placed to the sides of each eye and above and below one eye. When an eye movement is made by the participant the signal is large (in the hundreds of microvolts) as compared to the signals generated by the brain (typically 50 microvolts or less) and interferes with those brain-generated potentials. This interference is commonly called an EOG artifact or eye-movement artifact (Gratton, 1998). The inherent problem is that eye movements are very frequent and often occur at or around the critical time when participants are responding to task-relevant stimuli. Thus, if an EOG artifact occurs simultaneously with the critical potentials a researcher wishes to study (e.g. P300 or N400) it contaminates the physiological data that is recorded and vital for analysis.

Eye movement problems become even more troublesome when a researcher measures ERPs in divided visual field studies (DVF). In the DVF paradigm, where the participant is asked to fixate their eyes on a cross or prime in the center of a computer screen, it is very difficult for them to keep attending to that cross when they are constantly being presented with new stimuli in the left and right visual fields. As 
a result, participants have a hard time keeping their gaze on the center of the display and the presentation of the peripheral stimuli will often cause participants to shift their eyes left or right, which causes a large number of horizontal eye movements to appear in the data. While it might be easier to remove the "corrupted" data points, the elimination of a large amount of data is troublesome in terms of the a study's power and a real possibility that the smaller amount of clean data points remaining not containing eye movements may not be an accurate reflection of the true population (Gratton, 1998). It may even be the case that these horizontal eye movements do not occur randomly but may, in fact, be more frequent in particular conditions (i.e. more eye movements for trials in the left visual field compared to those in the right visual field). Keeping all of this in mind, researchers have two realistic choices: (1) find a way to correct for the eye movements in the data after the data has been collected or (2) come up with a way to present the stimuli that reduces or eliminates a participant's tendency (intentional or unintentional) to make horizontal eye movements.

Current methods used to prevent participants from making horizontal eye movements are very basic, and mostly ineffective. The most common technique, and least effective, used to eliminate eye movements is to instruct the participant to fixate and attend only to the fixation cross and/or prime in the center of the screen, while making sure not to look at the stimuli that will be presented to their left and/or right visual fields. Even if participants seem to follow instructions and maintain their focus 
on the center of the display they often vary their fixation points, and in many cases do not even fixate in the instructed center (Patching \& Jordan, 1998).

An additional method to ensure central fixation is to display a letter or number at the fixation point (versus a cross or an "X") and ask the participants to report that letter or number in addition to responding to the target stimuli (Bourne, 2006). On the trials in which the participants incorrectly report that letter or number, it is assumed that they were not centrally fixated and that trial is eliminated from the data set. This technique has been used in a number of studies, such as the work done by Belger \& Banich (1998) and Luh \& Levy (1995) (both cited in Bourne, 2006), but two issues have been documented (Jordan, Patching, \& Milner, 1998). For one, the assumption that central fixation is held only if the participants correctly report the letter or number can be easily confounded. If participants can respond to the peripheral targets, they should be able to do the same for the fixation stimuli (with possibly better performance due to the simplicity of the reporting task compared with the actual target task). Additionally, by asking the participants to report a letter or number in addition to the main task (i.e. lexical decision) they are forced into a dual task situation, which may affect the results of the main task by interfering with their responses to the actual target stimuli.

A third technique is for the experimenter to be present in the room with the participants and record the participants' eye locations and fixations, discarding any trials on which the participant has made an eye movement. Several studies, such as Deruelle \& de Schonen (1998) and Marzi \& Berlucchi (1977) (both cited in Bourne, 
2006) have used this method but there are inherent problems with this approach. The largest issue is the subjective nature of the observations of the participants' eye movements by an observer, who has to be vigilant throughout the experimental session. It is very difficult for an outside observer to accurately assess where another person is precisely fixated upon and whether that coincides with the proper fixation location (Gratton, 1998; Bourne, 2006). This observation becomes increasingly problematic the longer the session lasts, with any sudden loss or change in attention possibly occurring during a trial in which the participant moves his or her eyes. Additionally, there are other possible confounds such as demand characteristics, distracting the participants from the study by creating a sort of test anxiety situation where they may be more worried about pleasing the researcher then actually focusing their attention on the task at hand.

While the aforementioned techniques seem crude, more sophisticated approaches have emerged. One of the most popular is the use of eye-trackers that monitor the participants' eye movements while they are in the experimental session, replacing the need for the presence of an observer (Patching \& Jordan, 1998). The eye-trackers are significantly more accurate at identifying when participants move their eyes and, thus, making it easier to decide what trials to throw out. They also can give feedback to the participants during the session, indicating when they are not fixated on the center of the screen (Patching \& Jordan, 1998). The drawbacks, however, are that eye trackers are expensive and in constant need of re-calibration for 
each participant run, while also not solving the problem of preventing eye movements in the first place and still resulting in the loss of data.

A final solution, and one that is used primarily by ERP researchers, are to correct the contaminated data containing eye movements using sophisticated mathematical models (e.g. Gratton, 1998). These techniques can be done on-line (while the participants are running) or off-line, with the off-line methods being more sophisticated and more effective (Gratton, 1998). All mathematical corrections are based on the general model that the observed EEG value at any electrode is the sum of the uncontaminated EEG, the effects of the vertical EOG, and the effects of the horizontal EOG. To find the uncontaminated EEG value, one subtracts the sum of some scaled values of the horizontal and vertical EOG artifacts from the observed EEG value. These techniques can involve both frequency and time domain assumptions. The time domain techniques assume that the propogation of the EOG artifact from their source all the way to the back of the head is a nearly instantaneous process (e.g. Gratton, Coles, \& Donchin, 1983), whereas the assumption underlying the frequency domain techniques is that the scaling factors representing the EOG field propogation vary with the frequency and/or phase of the EOG at different electrode locations (Gratton, 1998). No matter the technique used and/or how sophisticated the models may be, however, the main problem is still not addressed because the corrections simply try to salvage the data with the EOG artifacts. They are not $100 \%$ effective, each has several assumptions underlying them, and there is always the worry that the corrected data still is not true to the actual population data. 
With all of the aforementioned techniques used to deal with the eye movement problem are commonly accepted in the field, they do not inherently solve the problem because all of these methods result in some loss of data. A potentially more advantageous solution is to present the stimuli to the participants using a paradigm that effectively eliminates the tendency for people to move their eyes (covertly or overtly). If such a paradigm can be designed the problem is solved at its very source and there is no need to either take preventative measures (instruct participants not to move their eyes or direct observation of eye movements) or to remove EOG in a postacquisition stage (with a mathematical model or the use of an eye tracker).

Information on how to reduce eye movements can be learned from the attention literature, with researchers examining such topics as what captures a person's attention in the visual environment and how eye movements and attention interact. Theories and techniques derived from the attention domain for both stimulus-driven and goal-driven attentional capture seem to provide one method for influencing the likelihood of eye movements (e.g. Atchley, Kramer, \& Hillstrom, 2000; Godijn \& Theeuwes, 2002; Jonides \& Yantis, 1988; Theeuwes, 1995; Theeuwes, Kramer, Hahn, \& Irwin, 1998; Theeuwes, Kramer, \& Kingstone, 2004; Yantis, 1993; Yantis \& Egeth, 1994; Yantis \& Hillstrom, 1994; Yantis \& Jonides, 1984, 1990). Ideas regarding the role of attention in driving eye movements are reviewed below.

\section{Stimulus-driven and Goal-driven Attentional Capture}

Within the attention domain, two types of selective attentional capture have been identified and examined closely: stimulus-driven (e.g. Atchley, Kramer, \& 
Hillstrom, 2000; Theeuwes, 1991; Yantis \& Jonides, 1984) and goal-driven (e.g. Folk, Remington, \& Johnston, 1992). Stimulus-driven, or bottom-up, selective attention occurs when an observer's attention is attracted via a property of an object that is independent of that person's goals or prior knowledge (van Zoest, Donk, \& Theeuwes, 2004). An everyday example would be a person walking down a sidewalk and looking straight ahead, but suddenly turns their head when a car pulls out of the driveway they are walking toward. Goal-driven or top-down attention occurs when the observer directs their attention based on knowledge about that stimulus and its attributes (van Zoest, Donk, \& Theeuwes, 2004). This could occur if one is looking for their silver car in a large parking lot and turns their head whenever a silver car appears in the visual scene. Aside from those real-world examples, many laboratory studies have examined what captures a person's attention in terms of stimulus-driven and goal-driven mechanisms.

The prevailing finding in the stimulus-driven capture literature is that attention is captured best when stimuli suddenly appear, or onset, in the visual scene (Atchley, Kramer, \& Hillstrom, 2000; Enns, Austen, Di Lollo, Rauschenberger, \& Yantis, 2001; Godijn \& Theeuwes, 2002; Jonides \& Yantis, 1988; Theeuwes, 1995;

Theeuwes, Kramer, Hahn, \& Irwin, 1998; Theeuwes, Kramer, \& Kingstone, 2004; Yantis, 1993; Yantis \& Egeth, 1994; Yantis \& Hillstrom, 1994; Yantis \& Jonides, 1984, 1990). This phenomenon is commonly referred to as the onset advantage. A main example of the research supporting the concept of onset advantage is Yantis and Jonides' (1984) demonstration via a modified procedure of the paradigm created by 
Todd and Van Gelder (1979), which distinguished participants' response between onset and no-onset (commonly referred to as offset) targets. Yantis and Jonides (1984) used simple figure-eight placeholders, situated around a central fixation point, whose line segments could be removed (or offset) to reveal simple letters such as $\mathrm{P}$, E, U, or S. This was coupled with the sudden onset of an additional letter in the upper right quadrant of the visual display. Participants were asked to determine which of the letters (the three offset and the one sudden onset) was the target letter that was shown at the beginning of the trial. Using reaction time (RT) as the dependent measure, participants responded faster to a target that was onset in the visual scene compared to one that was offset (camouflaged) from one of the figureeight placeholders.

Continuing with the argument that onsets capture attention in a stimulusdriven manner, subsequent work focused on what salient properties of the onset targets cause them to capture attention more effectively than offsets. Jonides and Yantis (1988) further refined their original paradigm comparing onsets to offsets, including two conditions that tested whether the change in luminance or a difference in color for the onsets (compared to the distractors) caused the onset advantage. In the color condition the target was a different color than the remaining letters (red target among green distractors for half the subjects and vice versa for the other half), while in the brightness condition the target was brighter than the others. Participants' RT were not significantly altered in either the brightness or color conditions, however responses were again slower to the offset targets. Yantis and Hillstrom (1994) used 
equiluminant stimuli to examine other possible salient properties that might capture attention. The study altered the targets' (whether offset or onset) texture, motion, and depth (binocular disparity) compared to the distractors. In addition, they defined the onset or offset targets as either old (offset of object in an occupied location to reveal target) or new (abrupt onset object in a new location). Again, participants responded to the onset (new) targets faster than the offset (old) targets across all conditions. The results lead toward a model of attentional capture stating that the onset advantage occurs due to the appearance of a new perceptual object in the visual scene (Yantis \& Hillstrom, 1994; Yantis \& Jonides, 1996).

Other researchers argue that the luminance change created by the new objects (abrupt onsets) is still an important stimulus-driven mechanism behind the attentional capture. Theeuwes (1995) argued that luminance change factors into attentional capture for onsets, using a different paradigm from Yantis and colleagues. Instead of figure-eight placeholders and having participants look for onset and offset targets, the objective was to find a line segment (horizontal or vertical) in a specific orientation (placed within a circle). Experiments were conducted in which either the color (isoluminant change) or luminance (no color change) of the target circle or segment were altered compared with the distractor segments within circles. Results showed that the abrupt luminance changes affected response performance, while the abrupt color changes did not. This work supports Yantis and Jonides' (1988) claim that color changes do not capture attention effectively, but also counters with the claim that luminance does have an effect on attentional capture. 
While Yantis's and Theeuwes's work demonstrate that stimulus-driven mechanisms seem to be behind the onset advantage in capturing attention, some researchers have argued that many goal-driven processes can be involved to override that advantage and help participants effectively ignore the onset stimuli (targets and/or distractors). Folk and colleagues $(1992,1994)$ argue that capture of a stimulus is contingent upon the target sharing features that are relevant to the task. In this way, it is not the onset distractor suddenly appearing in the display that captures a person's attention, it is that the distractor has identical properties to the target and agrees with the "current control settings" that were set in the system prior to seeing the stimuli (Folk, Remington, \& Johnston, 1992). Watson and Humphreys (1997) supported the notion of underlying goal-driven mechanisms using the aforementioned preview paradigm. Participants were able to prioritize the blue elements (new, onset stimuli) over the green elements (old stimuli) better if they were shown the display without the preview, demonstrating that the visual system can prioritize multiple new objects at the expense of old objects. These researchers have, thus, argued that observers can actively inhibit (a process that can be voluntarily switched on or off) the locations or properties of the old objects so as not to compete with the new objects.

Despite the aforementioned evidence for an influence of goal-driven mechanisms on the onset advantage in capturing attention, more work seems to demonstrate that stimulus-driven processes are more influential when observers are shown displays with new and old items. Returning to work by Theeuwes and colleagues, Donk and Theeuwes' (2001; 2003) used Watson and Humphrey’s (1997) 
preview paradigm and argued for an attention capture model supporting a bottom-up mechanism. Their conclusion was that search performance for the new elements (blue H's) was independent of the number of old elements displayed (blue A's and green H's). They argued that the new elements created a perceptually different luminance change from the old elements, and were, thus, prioritized over the old elements. Additional experiments using equiluminant stimuli (Donk \& Theeuwes, 2003) demonstrated that observers are quicker to respond to onset targets among new elements whether they appeared (1) equally among a set of old elements or new elements, (2) in varied proportions, (3) or even more among old than new. While these findings seem to add to Theeuwes' luminance argument, it also lends more weight to the new object hypothesis from Yantis' work. Further clarification is then needed to separate out which aspect is more critical.

While all of the previous work mentioned is built upon behavioral data, converging evidence for the onset advantage is presented in the cognitive neuroscience domain. Humphreys, Olivers, and Yoon (2006) worked with patients who all suffered from unilateral parietal damage. Damage to this region (especially when located in the right hemisphere) can lead to a variety of deficits, typically including a reduced sensitivity to stimuli in the region of space contralateral to the damage (also referred to as hemineglect). Humphreys and colleagues $(1993,1994)$ demonstrated that visual search tasks, particularly when the saliency of the targets was altered, for this patient group are abnormally difficult (as cited in Humphreys et al., 2006). When presented with figure-eight placeholder arrays of various sizes, 
targets were either offset from the placeholders or onset in an unoccupied region. The advantage for the onset targets was present whether or not they were shown in the ipsilateral or contralateral (in relation to the lesion) visual field and was equivalent to the data from age-matched controls. This demonstrates the robustness of the onset advantage with onsets still more powerful in capturing attention than offsets, even in affected visual regions of lesion patients.

Work using electrophysiological methods also lends support the unique ability of onsets to capture attention. Hickey, McDonald, and Theeuwes (2006) studied the onset advantage by examining an ERP component called the N2pc, which is believed to reflect attentional selection of an item via suppression of another (e.g Luck, Girelli, McDermott, \& Ford, 1997; Luck \& Hillyard, 1994; as cited in Hickey et al., 2006). Using a lateralized presentation paradigm from Theeuwes's previous work (1991), the N2pc waveform results demonstrate that even when the onset distractor was in the opposite visual field from the target participants still focused on the distractor before shifting their attention to the target. This work shows evidence of the attentional priority given to onsets at the level of actual brain mechanisms and lends further support to the behavioral work. All the mounting evidence explains what types of stimuli capture attention best (large luminance changes and new objects), but when discussing attentional capture there are other aspects of behavior that need to be reviewed before a complete picture is established. The main issue of this study is the reduction of horizontal eye movements, so the question becomes what connections are there between eye movements and attentional capture. 


\section{Eye Movements and Attentional Capture}

Next we turn to a discussion of the relationship between attentional capture and the motor execution of eye movements. An increasing number of studies have used eye trackers to examine the interplay between attention and eye movements. The execution and measurement of eye movements can not only confirm the reaction time data derived from attention research paradigms, but also increase our understanding of mechanisms which control the allocation of attention. Eye movements are interesting to researchers because evidence suggests that attention precedes the actual eye movement, or saccade, (e.g. Hoffman \& Subramanium, 1995) thus when attention can be controlled or diverted, a person's saccade provides a correlated behavioral indicator of this attentional shift.

Due to this connection, one can examine the two different types of saccades that are executed by an observer in conjunction with either stimulus or goal-driven attentional mechanisms. When sudden onset of a stimulus occurs in the periphery of the visual field the neurons in the magnocellular (M) visual pathways become activated. This causes the observer's attention to shift in a stimulus-driven fashion and their eyes make a rapid shift toward the stimulus. These rapid eye movements have the effect of completely changing what the observer is looking at and making them orient and draw attention from a previous area of interest to an entirely new one. Rapid shifts of the eyes are referred to as rapid or express saccades and they are known to occur between 180 and $200 \mathrm{~ms}$ after the onset of the stimulus (Becker, 1989). Goal-driven mechanisms can also cause saccades to stimuli in the visual field 
that are distinctly different from rapid saccades. These eye movements are as sudden as rapid saccades and are directed at stimuli in the visual scene that the observer finds interesting and/or wishes to get a more detailed or improved holistic view of. Goaldriven eye movements are called voluntary saccades and occur around $200 \mathrm{~ms}$ after a stimulus is presented (Becker, 1989).

Due to our growing knowledge about saccades and their sources, researchers have uncovered more findings regarding attentional capture using eye tracking technology. Supporting stimulus-driven mechanisms and their ability to override goal-driven processes, Theeuwes, Kramer, Hahn, and Irwin (1998) showed that a sudden onset stimulus both captures attention and causes observers to execute a saccade to that location even if the object (a color singleton) is irrelevant to the target and/or not where they intended to gaze. Theeuwes, Kramer, Hahn, Irwin, and Zelinksy (1999) furthered this work by demonstrating that not only do saccades shift (Experiment 1) toward the onset distractor first (about one third of the trials) but so does an observer's spatial attention (Experiment 2). These findings coincide with Hickey and colleagues' (2006) findings, discussed earlier, regarding the effectiveness of onsets to capture attention and alter brain activity. Additionally, these results converge with previous evidence presented and points toward onsets capturing attention and altering processing at multiple levels: brain activity, behavioral response, and oculomotor response.

Other studies measured saccades to help further refine what aspects of the onset advantage are more important: the luminance transient or the appearance of a 
new object. Irwin and colleagues (2000) demonstrated that the appearance of a new object is the more effective property for capturing attention than luminance. The distinction between the two was demonstrated using a bilateral array of circles enclosing figure-eight letters. The figure-eight placeholders (e.g. Yantis \& Jonides, 1984) were changed to the target or distractor letters and the enclosing circles that could differ via luminance or color (e.g. Theeuwes, 1995). In Experiment 3 one condition had participants responding to a target letter appearing inside a circle that changed luminance with an onset distractor (new circle and letter), while the second condition asked participants to identify a target inside an onset circle with a luminance distractor. Results showed that the onset distractor was still more effective both behaviorally and physiologically (more reflexive saccades occurring toward the distractor first than the target) than the luminance distractor. Further supporting the new object hypothesis, Boot, Kramer, and Peterson (2005) used the same paradigm as Irwin and colleagues (2000) and simply compared onsets (new) with offsets (old). The distractor was either onset or offset from the display in addition to the target (specified letter). Whether the distractors were shown via a color change, contour change, or luminance change, the onsets again had the advantage over offsets (faster RT and more saccades) in capturing attention. These results lead one to conclude that while luminance can capture attention, the appearance of a new object is more salient and, thus, more effective in capturing attention. This is especially true when stimuli appear in an empty location rather than appearing in a previously occupied location (i.e. onsets vs. offsets). 


\section{Possible Solution to the Problem}

All of the evidence from the onset advantage suggests that while onsets and offsets can create the same sensory changes in the environment, they do not appear to capture attention in the same way and, thus, do not share the same status in the visual system. It seems that an observer shows preferential attention for a stimulus that suddenly appears in the visual scene rather than appearing from behind something disappearing or disappearing altogether (e.g. Cole, Kentridge, Gellatly, \& Heywood, 2003; Samuel \& Weiner, 2001). This preference can be seen both behaviorally and physiologically. More importantly, this data regarding attentional preference for new items helps to explain why we see many horizontal saccades during divided visual field studies (DVF).

With all of the current problems that arise because of eye movements and the lack of completely successful correction techniques in DVF studies, a possible way to correct these problems is to apply the principles derived from the attention research reviewed above. In other words, researchers might find a way to present the stimuli without creating a situation in which the observer will involuntarily shift their attention and execute a reflexive saccade to the left or right visual fields. If the stimuli are revealed in an offset manner (created from old elements) rather than suddenly appearing as a new element in the display, the lateralized stimuli should not be as likely to cause a saccade to occur. This idea should not only work with simple targets, such as shapes and letters, but more complex ones like words and sentences. 
In the following studies, offset stimuli are applied during a DVF paradigm to help control the degree of rapid attentional capture and shifting generated by the lateralized targets. Both behavioral responses and eye movement data are recorded to determine whether or not the use of offset stimuli can reduce horizontal eye movements. One result of this manipulation is predicted to be the effective reduction of the horizontal EOG artifact. The predicted reduction in eye movements would support the findings in the attention literature (e.g. Boot, Kramer, \& Peterson, 2005; Irwin, Colcombe, Kramer, \& Hahn, 2000; Theeuwes, Kramer, Hahn, \& Irwin, 1998; Theeuwes, Kramer, Hahn, Irwin, \& Zelinsky, 1999).

\section{Experiment 1}

\section{Introduction}

The present study investigated the possibility of reducing horizontal eye movements in a divided visual field task through the use of an offset masking procedure. In this procedure, a bilateral mask is presented with the central prime that is then removed to reveal the lateralized target. Once the target is presented it is replaced by the same bilateral mask. The offset procedure contrasts with the typical onset procedure used in divided visual field studies which typically presents the participants with a bilateral mask after the target is presented (at which time a response is made by the participants). According to findings in the attention literature (e.g. Atchley, Kramer, \& Hillstrom, 2000; Jonides \& Yantis, 1988; Yantis, 1993; Yantis \& Egeth, 1994; Yantis \& Hillstrom, 1994; Yantis \& Jonides, 1984, 1990), 
displaying the targets in an offset manner (with the targets being revealed from a previously occupied location) instead of suddenly appearing in a previously unoccupied location (in an onset manner) should reduce the targets attentional priority. By reducing the attentional priority of the targets this should allow participants to more effectively inhibit reflexive saccades to the target location.

In addition to the primary goal of reducing horizontal saccades, the present study aimed to determine whether or not the new offset procedure would disrupt the robust language effects found in divided visual field studies. These robust effects include (1) the left hemisphere advantage over the right hemisphere in reaction time and accuracy for the target words and (2) more accuracy and quicker reaction times for target words that are related to the primes than those that are unrelated (see Chiarello, 1988 for review). In order to test for these, effects a lexical decision task is used, in which lexically ambiguous prime words (e.g. river) are presented centrally and are followed by the presentation of target words that are either semantically related (e.g. bank) or unrelated to the prime (e.g. dog) or non-word letter strings.

\section{Method}

\section{Participants}

Twenty-three participants from the University of Kansas undergraduate introductory psychology courses participated for course credit. All were righthanded, native English speakers, and had normal or corrected-to-normal vision. None of the participants had previously participated in an experiment similar to the current study. 


\section{Apparatus}

The primes, targets, and mask were presented onto a LCD color monitor placed $51.0 \mathrm{~cm}$ from the subject and connected to an IBM compatible personal computer. The stimuli were compiled and then presented via Neuroscan 4.2 STIM system software. Response times were measured to the nearest ms from the onset of the target to the pressing one of two keys on a STIM four-button keypad.

Eye movements were recorded through Neuroscan $\mathrm{Ag}-\mathrm{AgCl}$ electrodes that were connected to a Neuroscan amplifier. Electrodes were placed above and below the left eye, as well as to the left of the left eye and to the right of the right eye, in order to record the EOG signals that occur if subjects blinked and/or made a horizontal eye movement.

\section{Stimuli}

A practice list and four experimental lists were created. Each experimental list contained two blocks, one block using the new offset masking technique and the other having the standard onset presentation. The four lists were counterbalanced and coded for masking technique, visual field presentation of the words, semantic relatedness of the targets to the primes, and dominant and subordinate meanings of the targets to the primes. The words in the lists were taken from a previous study (Atchley, Burgess, \& Keeney, 1999) in which 320 ambiguous words were used as primes and the other half were words that either were related to their matched prime (RELISH-PICKLE), unrelated to their matched prime (RELISH-FLAVOR), or were pronounceable non-words (TACK-METHER). The practice list consisted of 25 
prime-target pairs and the experimental lists contained 160 prime-target pairs. The target words had a median letter length of 4.9 with a standard deviation of 1.19 . The targets were randomly assigned to either the left or right visual field, with an equal number of words being displayed to each visual field.

The mask consisted of two sets of seven identical checkerboard rectangles (length: $1.0 \mathrm{~cm}$; height: $2.5 \mathrm{~cm}$ ), each $3.0 \mathrm{~mm}$ apart, were placed $3.1 \mathrm{~cm}$ from the left and right of the fixation cross. The rectangles acted as perceived placeholders for the letters in the target words that would later appear on the screen (Figure 1).

Figure 1. The mask and procedure used for both the offset and the onset conditions.

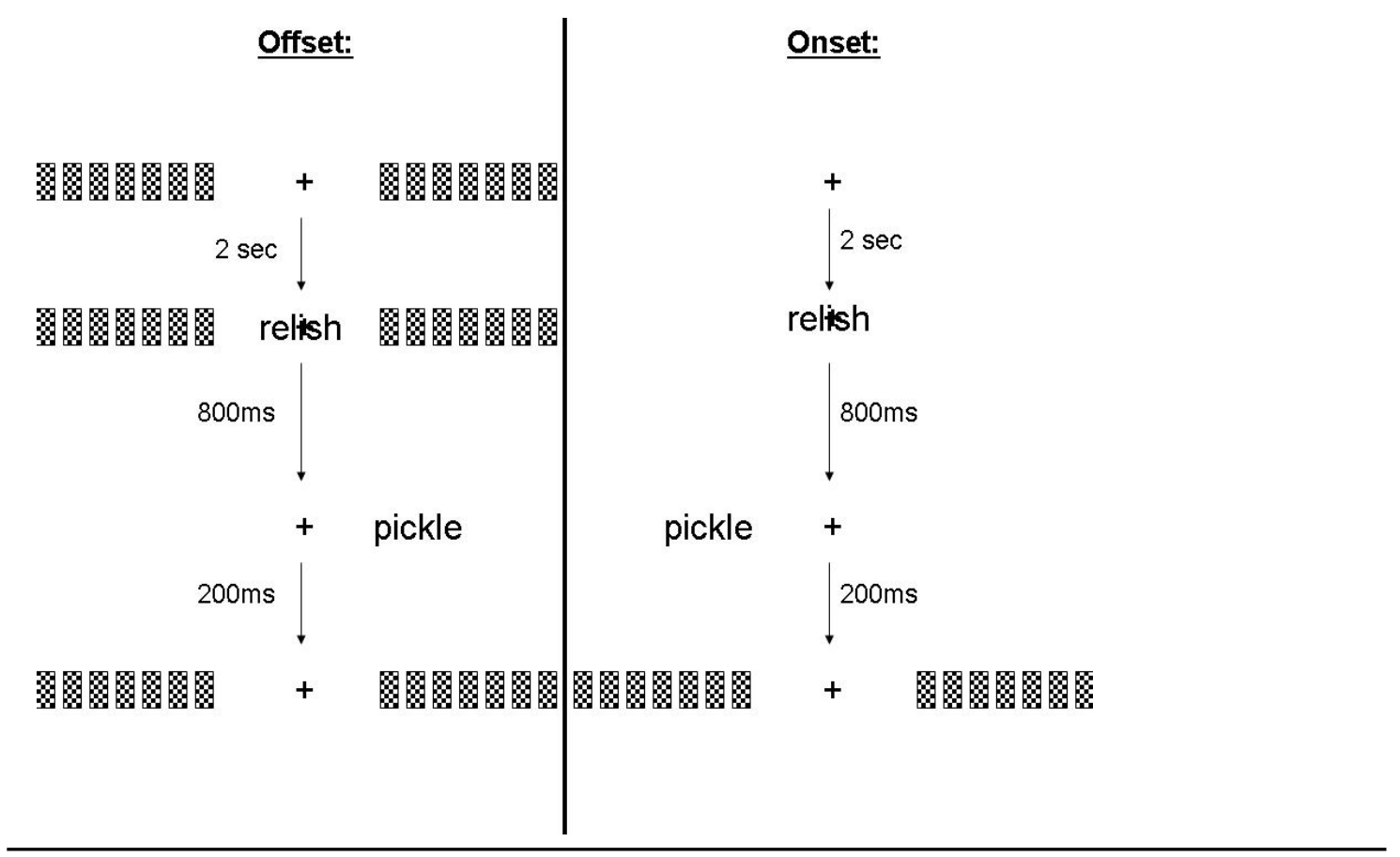

\section{Design and Procedure}

The design of the experiment was a 2 (masking type: offset and onset) x 2 (visual field of targets: LVF and RVF) x 2 (word type: word and non-word targets) $\mathrm{x}$ 
2 (prime target semantic relatedness) $\times 2$ (prime target subtype: dominant and subordinate) mixed factorial design. All the variables were presented within-subjects, with the masking type variable presented in separate blocks and the other four variables within each block. Two behavioral dependent variables were measured: reaction time and response accuracy. Two physiological dependent variables were also measured: number of eye movements made and the latency of the onset of each eye movement.

Each participant was tested individually in a dimly lit room, with an experimenter seated nearby. Participants placed their heads into a chin rest that was approximately $51 \mathrm{~cm}$ from the presentation monitor. The targets were presented approximately $4.5 \mathrm{~cm}$ from the fixation cross so as to fit the mask that was presented prior to their onset, with an approximate horizontal visual angle of 3.48 degrees.

For the target detection task, the participants first saw a white centrally located fixation cross immediately followed by the mask that was presented for 2 seconds. A prime was then displayed (with the mask still present) at a central location, directly over the fixation cross, for $800 \mathrm{~ms}$. Both the mask and the prime then offset and were immediately followed by the onset of the target word, which was presented for $200 \mathrm{~ms}$. The targets offset and were immediately followed by the presentation of the mask for two seconds, during which the participants made their response.

The onset condition had the participants look at the same white, central, fixation cross for two seconds. The prime, without the mask, followed and was 
displayed in the same central location as the fixation cross for $800 \mathrm{~ms}$. The prime then offset and was followed by the lateralized onset of the target, which was presented for $200 \mathrm{~ms}$ and immediately followed by the same mask used in the offset condition (Figure 1). The participants responded at that time.

Once the target was presented and then offset, the participants were asked to respond as to whether they believed the target presented was a word or a non-word. Responses were recorded on the STIM keypad for both accuracy and response time. The practice trial took approximately five minutes and the experimental trial lasted about 30 minutes, with total session time lasting approximately one hour.

\section{Results}

\section{Behavioral}

A Repeated Measures ANOVA was performed on participants' mean accuracy per condition with mask type, visual field of target presentation, and relatedness of the prime-target pairs as factors. There were three significant main effects for mask type, $\mathrm{F}(1,22)=10.10, \mathrm{p}<.01, \mathrm{MS}_{\text {error }}=.02$; visual field, $\mathrm{F}(1,22)=$ $6.41, \mathrm{p}=.02, \mathrm{MS}_{\text {error }}=.03 ;$ and relatedness, $\mathrm{F}(1,22)=5.16, \mathrm{p}=.03, \mathrm{MS}_{\text {error }}=.07$ There were no significant interactions present.

Overall accuracy for all trials was 66\% (.03). For the mask type, onsets had higher accuracies than offsets, Onsets: 70\% (.03); Offsets: 63\% (.03). There was an advantage for the left hemisphere over the right hemisphere, LH/RVF: 70\% (.03); RH/LVF: $63 \%$ (.03). Related trials were more accurate than unrelated trials, Related: 68\% (.03); Unrelated: 64\% (.03) 
Number of Eye Movements

A Repeated Measures ANOVA was performed on the number of eye movements that participants made per condition. There was no significant effect for mask type $\mathrm{F}(1,22)=.38, \mathrm{p}=.55, \mathrm{MS}_{\text {error }}=12.14$; visual field, $\mathrm{F}(1,22)=.33, \mathrm{p}=.57$, $\mathrm{MS}_{\text {error }}=14.00$; or relatedness, $\mathrm{F}(1,22)=.32, \mathrm{p}=.58, \mathrm{MS}_{\text {error }}=2.90$. A significant three-way interaction was present between the three factors, $F(1,22)=5.71, p=.03$, $\mathrm{MS}_{\text {error }}=2.48$ (see Table 1). No other interactions were significant.

Examining the marginal means for the three-way interaction between mask, visual field, and relatedness, it appears that the differences occurred in the onset mask condition. When the target was presented to the left visual field/right hemisphere, participants seemed to make more eye movements in the unrelated condition than the related condition, Related: 5.17 (1.24); Unrelated: 5.696 (1.39). When targets were presented to the right visual field/left hemisphere, however, more eye movements were when the target was semantically related to the prime than when it was semantically unrelated, Related: 5.61 (1.32); Unrelated: 4.52 (1.24). Since these differences were small and our sample size was small for this experiment, a NeumanKeuls post-hoc comparison was run. None of the differences mentioned above were deemed reliable. 
Table 1.

Table 1

Mask x Visual Field x Relatedness Interaction for Eye Movements per Condition

\begin{tabular}{|c|c|c|c|c|}
\hline \multirow[b]{2}{*}{ Condition } & \multirow[b]{2}{*}{$M$} & \multirow[b]{2}{*}{$S D$} & \multicolumn{2}{|c|}{ 95\% Confidence Interval } \\
\hline & & & Lower Bound & Upper Bound \\
\hline \multicolumn{5}{|l|}{ Onset } \\
\hline \multicolumn{5}{|l|}{$\mathrm{LVF} / \mathrm{RH}$} \\
\hline Related & 5.17 & 1.24 & 2.60 & 7.75 \\
\hline Unrelated & 5.70 & 1.39 & 2.81 & 8.58 \\
\hline \multicolumn{5}{|l|}{$\mathrm{RVF} / \mathrm{LH}$} \\
\hline Related & 5.61 & 1.32 & 2.88 & 8.34 \\
\hline Unrelated & 4.52 & 1.24 & 1.96 & 7.09 \\
\hline \multicolumn{5}{|l|}{ Offset } \\
\hline \multicolumn{5}{|l|}{$\mathrm{LVF} / \mathrm{RH}$} \\
\hline Related & 5.22 & 1.26 & 2.60 & 7.84 \\
\hline Unrelated & 4.91 & 1.14 & 2.56 & 7.27 \\
\hline \multicolumn{5}{|l|}{$\mathrm{RVF} / \mathrm{LH}$} \\
\hline Related & 4.65 & 1.18 & 2.20 & 7.10 \\
\hline Unrelated & 4.96 & 1.11 & 2.64 & 7.27 \\
\hline
\end{tabular}

Eye Movement Latency

A Repeated Measures ANOVA was also performed on the mean latency of onset of the participants' saccades. The only significant main effect occurred for mask type, $\mathrm{F}(1,22)=44.82, \mathrm{p}<.01, \mathrm{MS}_{\text {error }}=567.02$. The participants initiated an eye movement earlier during the trials with the onset mask than during trials with the offset mask, Onset: 167.69 (4.79); Offset: 203.34 (3.54). No significant main effects 
were present for visual field, $\mathrm{F}(1,22)=.001, \mathrm{p}=.96, \mathrm{MS}_{\text {error }}=212.29$; or relatedness, $\mathrm{F}(1,22)=4.33, \mathrm{p}=.07, \mathrm{MS}_{\text {error }}=45.69$. No significant interactions were present.

\section{Discussion}

When examining the number of eye movements initiated per condition by the participants, the main goal was not achieved. Participants made the same number of eye movements during the offset procedure as the onset procedure. This finding contradicts the evidence from the attention literature because the act of revealing the target stimuli instead of it suddenly appearing did not seem to alter its attentional priority as reflected by lateralized eye movements. The one difference found between the two procedures was the latency of initiating the saccades. Saccades made during the offset procedure were initiated over $30 \mathrm{~ms}$ later than those during the onset procedure (203ms vs. $167 \mathrm{~ms})$. This difference suggests that the saccades made during the onset procedure were more reflexive in nature, responding to the sudden appearance of stimuli in one side of the visual field. The saccades in the offset procedure, however, might be explained as being programmed by the participants once they detect a task relevant word is present in one of the visual fields.

While the eye movement data did not support the primary a priori predictions, the behavioral data supported the secondary goal by demonstrating that the offset procedure still resulted in the robust language effects previously seen in the divided visual field literature. The left hemisphere advantage was present as was the advantage for related targets over unrelated targets. A significant issue with the behavioral data, however, was the low accuracy rates across all conditions. Normal 
accuracy rates for divided visual field studies are typically around 75\%-80\% (e.g. Atchley, Burgess, \& Keeney, 1999), but the mean accuracy rate for the present study was $66 \%$. While the accuracy for the onset condition (which closely resembles the typical paradigm used in DVF research) was closer to normal at 70\%, it is still lower than the desired range. This is a concern that should be monitored in subsequent experiments.

When examining the results, there are some concerns that should be discussed. First, only twenty-three subjects were run in this experiment. Given that the primary finding in this study is an unexpected null result, this being the equal number of eye movements in the onset and offset conditions, this relatively low number of participants must raise the concern that statistical power was insufficient. One thing, however, that might mitigate this concern is the finding that we had sufficient power in our design to observe a reliable difference in the latency of the eye movements, which one might expect to be a more "subtle" dependent variable. Another set of concerns we wish to raise involve procedural parameters. An a priori perceived difficulty of the task with the new offset procedure resulted in the lengthening of the presentation times for both the prime and target words to $800 \mathrm{~ms}$ and $200 \mathrm{~ms}$ respectively. This timing change pushes the acceptable limits in the literature and adds skepticism for the behavioral findings. Finally, the offset procedure used was rather simplistic. The mask used was not dynamic (using simple, checkerboard rectangular boxes which remained constant throughout instead of an ever changing pattern). Possibly the most important issue is that when the target was 
presented a drastic luminance change still occurs from one visual field compared to the other. This luminance change was due to the target being present in one visual field with nothing present in the opposite visual field. The change in luminance might attract participants' attention, causing them to intiate saccades in the offset procedure just as it would in the onset procedure. Experiment 2 was conducted in order to address these various methodological and statistical concerns.

\section{Experiment 2}

\section{Introduction}

The previous experiment examined the possibility of using an offset masking procedure to reduce horizontal eye movements while attempting to preserve the typical language effects seen in divided visual field studies. While the language effects were still present, the new masking procedure was not effective in reducing the number of eye movements made by the participants. We had some concerns, however, about the procedure and mask used in Experiment 1. The present study aimed to improve upon and test the behavioral effects of a new offset procedure and mask design while attempting to preserve the robust divided visual field language effects seen in the previous study.

In order to improve upon the mask and present the stimuli in a more dynamic fashion, two changes were implemented. The first change needed was in the design of the mask itself to make it an offset (as defined in the attention literature). The mask used in Experiment 1 was very simple and constant, with 
bilateral, letter-sized, and checkerboard patterned rectangular boxes used as the mask in all conditions. This is not ideal for obscuring a word before and after it is shown because the words and the mask are separate and unique items in a person's visual scene. No one would perceive that the target word was originally embedded in the boxes, which were then taken away to reveal the word. The target letters that Yantis and Jonides (1984) displayed were part of a figure eight in which line segments were removed (offset) to reveal them. In order to achieve a similar effect with target words, in our experiment we made the target words part of a jumble of other words or letter strings which are then offset to reveal the word of interest.

In addition to altering the nature of the mask, we were concerned that the luminance change, occurring in the opposite visual field of the target, also needed to be eliminated. If the luminance change in each visual field is nearly identical, there should be no attentional bias created; thus, people should not be able to use this change in luminance as a cue to orient their attention and, subsequently, their eyes to either visual field. This problem led to a second alteration in the design of the mask. In order to balance out the luminance change in both visual fields, the simplest solution would be to simultaneously add a non-word letter string on the opposite visual field of the target. This letter string would be considered a placeholder, or a filler, and would be present for each trial. Each placeholder would be equal in letterlength to the target, eliminating (as much as possible) the difference in luminance across both visual fields. Additionally, in order to ensure that the procedure and mask used would truly be dynamic throughout (and not prone to pattern detection by the 
participants) we also thought it important to have each target word paired with its own placeholder.

In addition to the two changes to the mask mentioned above, the presentation times of the prime and target words were reduced. Experiment 1 had longer than normal presentation times for both due to a priori considerations about the task difficulty, which also led to concerns for the results and their interpretation. For the present study those times were adjusted back to acceptable values for both sets of words, based on times commonly used in DVF studies (see Chiarello, 1988 for review).

Due to all the changes made to the mask and the procedure, two questions emerged for the study. The first is whether or not participants can still detect the target word given the presence of the non-word placeholder. To address this question we asked participants to indicate which visual field the target word appears in. A visual field detection task using word stimuli is unusual, but becomes vital to determine whether or not the placeholder has changed the attentional bias for the target word. If the attentional bias is affected by this luminance control, then detection of the words should not be biased to either visual field. If the attentional bias is unaffected, then a visual field main effect should occur. The prediction is that in the visual field detection task, participants should be able to detect the targets but show no attentional bias toward one visual field over the other.

The second question asks if the robust language effects found in the DVF literature and in the previous study can still be demonstrated. This is particularly 
important given that the task is now not the same lexical decision task used in Experiment 1 or in typical DVF research. Instead of being presented with one letter string and making a lexical judgment, participants see bilaterally presented letter strings and are asked to determine whether or not either one of those strings is a word. While the task is still a lexical decision, the complexity of the task is altered.

Participants now have to process two letter strings simultaneously (one in each visual field and, thus, one by each hemisphere). There is no doubt that this should be more difficult, so we expect accuracy rates to be lower than the normal 75-80\%. Even with lower accuracy rates, we still predict that participants can complete the task and the language main effects will be present.

Note that due to the nature of the questions being asked, only behavioral data needs to be collected. The original issue of controlling horizontal eye movements will again be addressed in Experiment 3.

\section{Method}

\section{Participants}

Forty-four participants from the University of Kansas undergraduate introductory psychology courses participated for course credit. All were native English speakers, right-handed, and had normal or corrected-to-normal vision. None of the participants participated in the previous study. The participants were each included in both the visual field detection task and the lexical decision task, which were conducted in one session. 


\section{Apparatus}

The primes, targets, and mask were presented onto a LCD color monitor placed $51.0 \mathrm{~cm}$ from the subject and connected to a Dell XPS personal computer. The stimuli were compiled and presented via E-Prime 1.1 software. Response accuracy was measured, in milliseconds, from the onset of the target to the pressing one of two keys on a keypad.

The change in software occurred due to a couple of important factors, the first being that the lab switched from STIM to E-Prime as the stimulus presentation software for ERP sessions. The other reason for the change was that the STIM software was limited in its design capabilities, not allowing for the dynamic mask we envisioned, which forced us to use a more crude design in the first experiment. Designing and programming in E-Prime also allowed for better control of the stimuli presentation, given the complexity and type of stimuli we used (allowing the targets to be presented for $185 \mathrm{~ms}$ rather than $200 \mathrm{~ms}$ ).

\section{Visual Field Detection Task}

Stimuli.

One practice list and four experimental lists were created. The lists were counterbalanced and coded for visual field presentation of the words and semantic relatedness of the targets to the primes. The practice list consisted of 15 prime-target pairs, with the experimental lists each containing 64 pairs. Target words had a median length of five letters, with a standard deviation of 1.38. The targets were 
randomly assigned to either the left or right visual field, with an equal number placed in each visual field.

\section{Design and procedure.}

The design of the experiment was a 2 (visual field of targets: LVF and RVF) $\mathrm{x}$ 2 (word type: word and non-word targets) x 2 (prime target semantic relatedness) mixed factorial design. All the variables were presented within-subjects. One behavioral dependent variable was measured: response accuracy. It was our expectation, based on results from Experiment 1, that overall accuracy would be too poor to allow for reaction time analyses. Each participant was tested individually in a lit room. Participants placed their heads onto a chin rest $51 \mathrm{~cm}$ in front of the presentation monitor.

The participants first saw a black centrally located fixation cross paired with both the target and the non-word placeholder (each on opposite visual fields). The target and placeholder were then instantly $(16 \mathrm{~ms}$ after their appearance, which is too fast for the participants to detect) covered by two identical non-word letter strings. This was displayed for one second followed by a central prime word presented (with the mask still present) directly over the fixation cross for $750 \mathrm{~ms}$. Then the prime and the mask were offset revealing both the target and the non-word placeholder, which were presented for $185 \mathrm{~ms}$. At that time the target and placeholder were then immediately covered up by the same identical two non-word letter strings (Figure 2). 
Figure 2. The new mask and the procedure for Experiment 2.

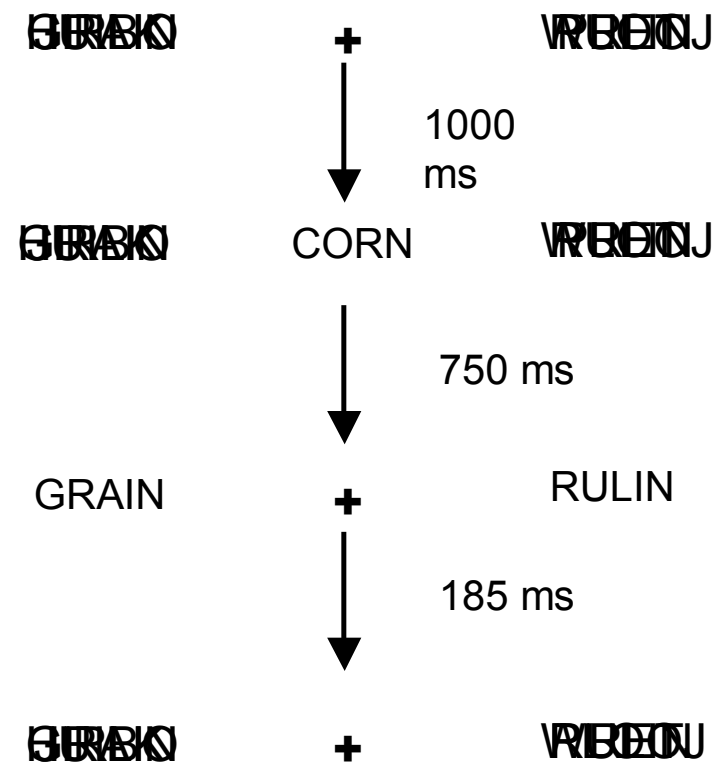

The participants' task was to detect (with explicit instructions not to move their eyes from the central fixation) which visual field the target was presented to. As soon as it was detected, the participants pushed either " 1 " or " 2 " on the numeric keypad with their right hand. "1"corresponded to the LVF, while "2" was for the RVF.

Each participant was given a practice list prior to running with the experimental list. Total running time for the session was approximately 15 minutes. Modified Lexical Decision Task

Stimuli.

Four experimental lists and one practice list were created. Each list was counterbalanced and coded for visual field presentation of the words and semantic relatedness of the targets to the primes. The practice list contained 15 prime-target 
pairs, while the experimental lists consisted of 128 pairs. The words were taken from the same source as Experiment 1 and, thus, have the same characteristics.

\section{Design and procedure.}

The design of the experiment was a 2 (visual field of targets: LVF and RVF) $\mathrm{x}$ 2 (word type: word and non-word targets) x 2 (prime target semantic relatedness) mixed factorial design. All the variables were presented within-subjects. Response accuracy was the only dependent variable measured.

The presentation of the stimuli was identical to that mentioned for the visual field detection task. For the task itself, participants were instructed to decide whether a real word was present or not when the target and placeholder were displayed. If a word was present then the participants were to press "1" on the keypad, and if no words were present then the participants were to press " 2. "

Each participant was given a practice list prior to running with the experimental list. Total running time for the session was approximately 15 minutes.

\section{Results}

It was observed in both tasks that a significant portion of participants had accuracies below $50 \%$, which would be the objective level of chance performance in this task. Ten participants (23\%) fell below 50\% accuracy in the visual field detection task, and eight participants (18\%) were below 50\% accuracy in the modified lexical decision task (see Table 2 for a more detailed breakdown). To check whether the inclusion of participants' responses significantly influenced the overall pattern of results, separate analyses were run on each task for (1) all the participants 
and (2) for participants with accuracies above 50\%. Four Repeated Measures ANOVAs were performed on participants' mean accuracies per condition, with visual field of target presentation and relatedness of the prime-target pairs as factors.

Table 2.

Table 2

Mean Accuracy Grouping for Participants in Experiment 2

\begin{tabular}{lcc}
\hline & \multicolumn{2}{c}{ Task } \\
\cline { 2 - 3 } Accuracy Range & Visual Field Detection & Modified Lexical Decision \\
\hline $75 \%+$ & 5 & 1 \\
$65-74 \%$ & 6 & 11 \\
$55-64 \%$ & 16 & 11 \\
$45-54 \%$ & 12 & 3 \\
$35-44 \%$ & 5 & 1 \\
$25-34 \%$ & 0 & 1 \\
$0-25 \%$ & 0 & 0 \\
\hline
\end{tabular}

\section{Visual Field Detection Task}

(1) Grand mean accuracy for all forty-four participants was 59\% (.02). There was a significant main effect for visual field, $\mathrm{F}(1,43)=5.14, \mathrm{p}=.03, \mathrm{MS}_{\text {error }}=.03$; with the left hemisphere more accurate than the right hemisphere, LH/RVF: $62 \%$ (.02); RH/LVF: 56\% (.02). No significant main effect for relatedness was present, $\mathrm{F}(1,43)=1.48, \mathrm{p}=.23, \mathrm{MS}_{\text {error }}=.01$. The interaction between visual field and relatedness was not significant, $\mathrm{F}(1,43)=.01, \mathrm{p}=.91, \mathrm{MS}_{\text {error }}=.01$.

(2) Ten out of the forty-four participants had a total mean accuracy below $50 \%$, leaving thirty four participants with an overall mean accuracy of $63 \%(.02)$. 
There were no main effects for visual field, $\mathrm{F}(1,33)=2.04, \mathrm{p}=.16, \mathrm{MS}_{\text {error }}=.03$; or relatedness, $\mathrm{F}(1,33)=.24, \mathrm{p}=.63, \mathrm{MS}_{\text {error }}=.01$. There was also no significant interaction, $\mathrm{F}(1,33)=.09, \mathrm{p}=.77, \mathrm{MS}_{\text {error }}=.01$.

\section{Lexical Decision Task}

(1) Grand mean accuracy for all participants was 58\% (.02). There were no significant main effects for visual field, $\mathrm{F}(1,43)=.65, \mathrm{p}=.43, \mathrm{MS}_{\text {error }}=.04$; or relatedness, $\mathrm{F}(1,43)=1.89, \mathrm{p}=.18, \mathrm{MS}_{\text {error }}=.02$. There was also no significant interaction, $\mathrm{F}(1,43)=.54, \mathrm{p}=.47, \mathrm{MS}_{\text {error }}=.01$.

(2) Eight out of the forty-four participants had a total mean accuracy below $50 \%$, leaving a modified mean accuracy of $61 \%(.01)$ for the remaining thirty-six participants. There were no significant main effects for visual field, $F(1,35)=.19$, $p$ $=.67, \mathrm{MS}_{\text {error }}=.04$; or relatedness, $\mathrm{F}(1,35)=.97, \mathrm{p}=.33, \mathrm{MS}_{\text {error }}=.02$. Additionally, no significant interaction was present, $\mathrm{F}(1,35)=.03, \mathrm{p}=.86, \mathrm{MS}_{\text {error }}=.01$.

\section{Discussion}

Before examining the results further, the low accuracy rates need to be

discussed. Twenty-three percent of the participants from the visual detection task had overall accuracy rates below 50\%, and $18 \%$ had overall accuracies below $50 \%$ in the modified lexical decision task (see Table 2 for a thorough breakdown). Not only does this cause a significant loss of subjects but even the participants who were not dropped had overall accuracies around 63\%, which is much lower than we generally see in this kind of research. This adds to the concern raised from Experiment 1 that 
these tasks are becoming increasingly more difficult for the participants as we change these attention related stimulus characteristics.

For the visual field detection task, a main effect for visual field of target presentation was present only when all forty-four participants were included. When the thirty-four participants who had accuracies above 50\% were analyzed, the main effect was not present. While this is the only effect affected by the change in sample size, it is a cause for concern and adds more speculation to the outcome and difficulty in the interpretation of the results. The interpretation from the visual detection task is that the addition (and luminance change neutralizer) of an equal length non-word placeholder, placed opposite of the target word alters attentional bias but that bias may not be entirely eliminated. This indicates that the placeholder, in some capacity, reduced the salience of the target word in capturing attention. There is, however, still a trend toward target words in the right visual field being easier to detect than the left visual field. Whether this reduction in attentional bias is strong enough to significantly affect the participants' horizontal eye movements, however, still needs to be addressed.

Regarding the modified lexical decision task, the results did not support the prediction that the same language main effects generally seen in DVF studies can be shown using the new mask and procedures. Both the visual field and relatedness main effects were eliminated in the current study, which is troubling and problematic. If the typical language effects in divided visual field studies cannot be replicated with the new mask and/or placeholder procedures, its usefulness in future research is 
jeopardized. Before this new paradigm is deemed ineffective for language studies, however, it must again be stated that although participants made a lexical decision, the new paradigm created here alters the nature of that decision. The addition of the placeholder opposite the target words creates a more complex task in which the participants must evaluate two letter strings simultaneously. That is a significant task difference from the typical procedure used in DVF language studies and raises questions on whether or not the results from the modified task can be compared on the same level as the usual lexical decision tasks from the literature. This task change issue will be addressed later on.

After examining the results, the next step is to determine what effect the changes made to the mask and procedure from Experiment 1 to Experiment 2 had on the DVF language effects. The first change was the use of the dynamic mask (that offset to reveal the targets), which is more similar to the no-onsets used in the attention literature (e.g Yantis \& Jonides, 1984) than the letterboxes previously used in Experiment 1. The second change was the addition of a equal-length letter string placeholder opposite of the target. Between the two, which is having a greater effect on the behavioral results? Additionally (and going back to the original issue in the study), do the new mask and placeholders affect attentional mechanisms to the point that they reduce horizontal eye movements? 


\section{Experiment 3}

\section{Introduction}

Experiment 3 attempted to answer two main questions. The first being which experimental manipulation to the mask paradigm in Experiment 2 is having a greater impact: the dynamic offset mask or the placeholder. The previous experiment used a newly designed offset mask and procedure and tested whether or not it affected participants' attentional bias, in addition to determining if the typical language effects found in DVF studies were affected. One positive outcome from Experiment 2 is that we have some evidence suggesting that the attentional bias might have been eliminated. However, through the simultaneous introduction of these two methodological changes, we are now seeing no reliable linguistic effects. Before this new paradigm is deemed ineffective in studying language, further examination of why the effects seen in Experiment 1 disappear in Experiment 2 is necessary.

In addition to examining the behavioral outcomes of the new mask and procedures, the original question raised throughout the study of whether or not horizontal eye movements can be reduced via on offset masking procedure is still vital. Now that a dynamic offset mask is being used, instead of the letterboxes from Experiment 1, it should succeed in affecting attentional capture; thus, reducing horizontal eye movements where the procedure in Experiment 1 failed. The use of the placeholder to eliminate the luminance contrast created by the target should also help reduce attentional capture and reduce horizontal eye movements. Concurrent to 
predicting a reduction in the number of horizontal eye movements, a replication of the latency effect from Experiment 1 is also expected.

\section{Method}

\section{Participants}

Thirty-three participants from the University of Kansas undergraduate introductory psychology courses participated for course credit. All were righthanded, native English speakers, and had normal or corrected-to-normal vision. None of the participants participated in the previous experiments.

Apparatus

The stimuli were presented onto a LCD color monitor placed $51.0 \mathrm{~cm}$ from the subject and connected to a Dell XPS personal computer. All the stimuli were compiled and presented via E-Prime 1.1 software. As in Experiment 1, Neuroscan $\mathrm{Ag}-\mathrm{AgCl}$ electrodes that were connected to a Neuroscan amplifier and recorded via Neuroscan 4.2 software. Electrodes were placed above and below the left eye, as well as to the left of the left eye and to the right of the right eye, in order to record the EOG signals that occur if subjects blinked and/or made a horizontal eye movement. Stimuli

Four experimental lists and two practice lists were created. Each list was counterbalanced and coded for the presence or absence of a non-word placeholder, visual field of target presentation, the semantic relatedness of the prime-target pairs, and the dominant and subordinate meanings of the targets to their primes. One practice list of 15 prime-target pairs and two experimental lists of 128 prime-target 
pairs were used for each block, with the order of presentation for each block and lists within each block counterbalanced. Again, the words were taken from the same source as Experiment 1, hence, the targets have the same characteristics.

\section{Design and Procedure}

The design of the experiment was a 2 (masking type: offset and onset) $x 2$ (placeholder: present and absent) x 2 (visual field of targets: LVF and RVF) x 2 (word type: word and non-word targets) x 2 (prime target semantic relatedness) mixed-factorial design. All the variables were presented within-subjects, with the masking type variable presented in separate blocks and the other five variables within each block. Response accuracy was the only behavioral dependent variable measured. Two physiological dependent variables were measured: number of eye movements made and the latency of the onset of each eye movement. Once again, each participant was tested in a lit room and placed their chin in a chin rest $51 \mathrm{~cm}$ away from the LCD monitor.

The offset mask block was similar to the setup mentioned in Experiment 2; however, half the trials in each block had no non-word placeholder present (along with its mask) in the opposite visual field as the target. For the onset mask block, the fixation cross was presented alone for one second. The central prime was then presented, followed by the target and its non-word placeholder on half the trials for $185 \mathrm{~ms}$. The same post-target mask is used for all four stimulus presentation conditions (see Figure 3). 
Figure 3. Masking and Placeholder conditions for Experiment 3.

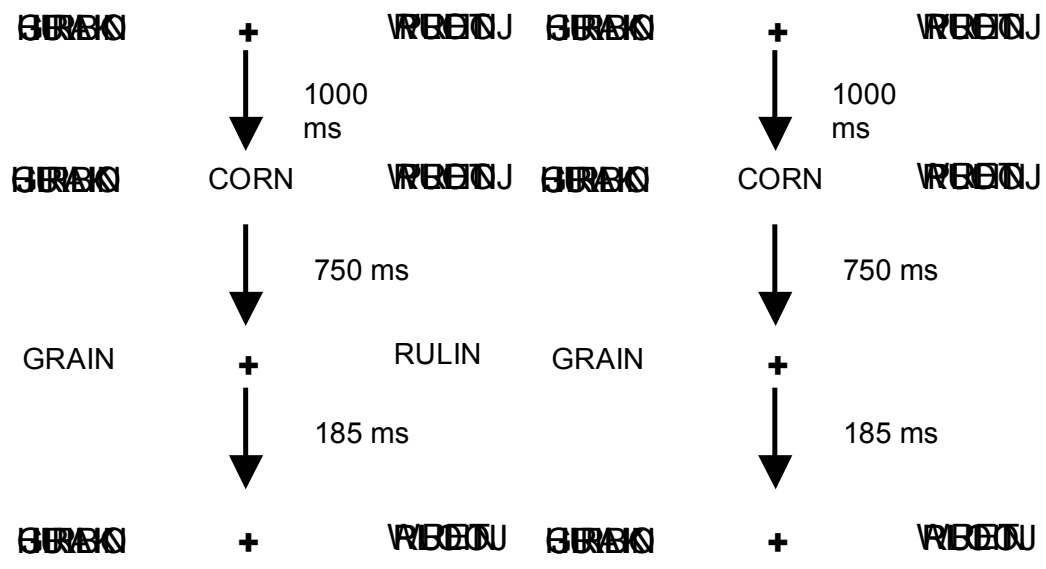

Offset with placeholder

Offset without placeholder

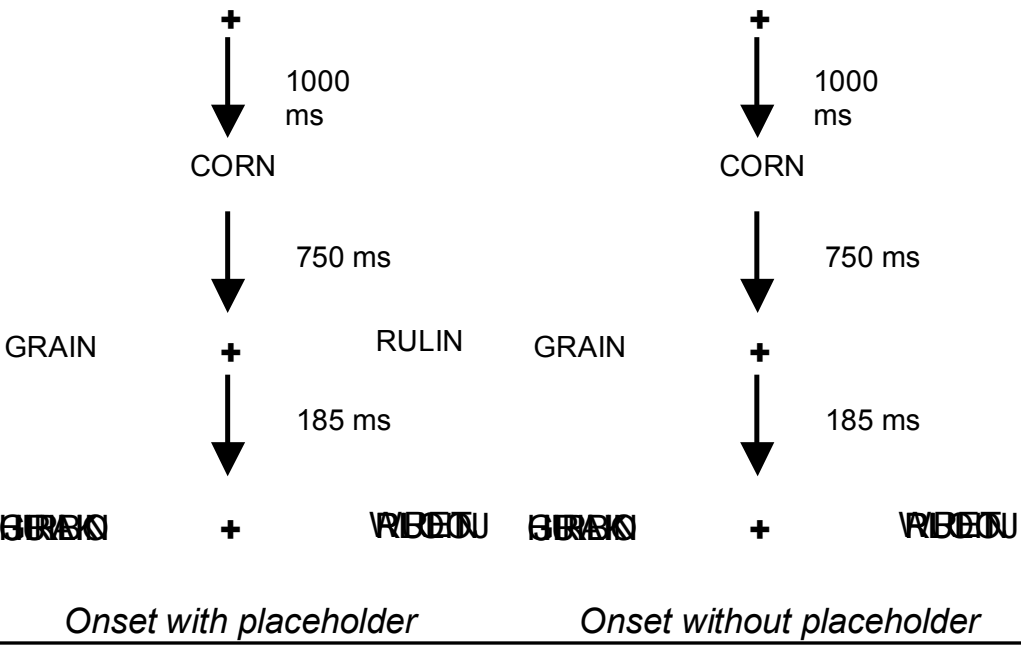

For both blocks, participants were to decide whether they saw a word either when only the target was present or when both the target and placeholder were displayed. "1" on the keypad was pressed if a word was present, while " 2 " was pressed if no word was present.

Both blocks were split up into two mini sessions, with each block lasting approximately 30 minutes. Each participant was given a practice list for each block. Total time for the setup and experimental session was approximately one hour. 


\section{Results}

Standard criteria for accuracy rates $(\sim 75 \%-80 \%)$ were not used due to the lower accuracy shown by participants throughout this series of experiments (see grand means for Experiments $1 \& 2$ ). To use the usual criteria would have meant a loss of data that might have affected our ability to generalize the results in regards to attentional processing. We wanted to be sure that we were not limiting the range of data being considered, recognizing that the behavioral results would necessarily be less comparable with DVF data found in the literature.

\section{Behavioral}

A Repeated Measures ANOVA was performed on the participants' mean accuracy per condition, with mask type, placeholder presence, visual field of target presentation, and relatedness of the prime-target pairs as factors. There was a significant main effect for mask, $\mathrm{F}(1,32)=5.00, \mathrm{p}=.03, \mathrm{MS}_{\text {error }}=.03$; visual field, $\mathrm{F}(1,32)=4.86, \mathrm{p}=.04, \mathrm{MS}_{\text {error }}=.10$; and relatedness, $\mathrm{F}(1,32)=6.21, \mathrm{p}=.02, \mathrm{MS}_{\text {error }}$ $=.02$. No significant main effect for the presence or absence of the placeholder occurred, $\mathrm{F}(1,32)=.85, \mathrm{p}=.36, \mathrm{MS}_{\text {error }}=.03$. No significant two-way interactions were present. There was one significant three-way interaction between placeholder, visual field, and relatedness, $\mathrm{F}(1,32)=10.16, \mathrm{p}<.01, \mathrm{MS}_{\text {error }}=.01$ (see Table 3 ).

For the mask main effect, onsets had higher accuracies than offset, Onset: 58\% (.02); Offset: 55\% (.01). The typical left hemisphere advantage was observed over the right hemisphere, LH/RVF: 59\% (.02); RH/LVF: 53\% (.02). Also, accuracy 
on related trials was higher than for unrelated trials, Related: 58\% (.01); Unrelated: $55 \%(.01)$.

A Neuman-Keuls post hoc comparison was run on the three-way interaction (see Figure 4). When the placeholder is absent, there is reliable priming in the left hemisphere but a trend (though not reliable) toward reverse priming in the right hemisphere. When the placeholder is present, however, there is no reliable priming in the left hemisphere (a trend is present) but priming does occur for the right hemisphere.

\section{Table 3.}

Table 3

Placeholder x Visual Field x Relatedness Interaction for Behavioral Accuracy in Experiment 3

\begin{tabular}{|c|c|c|c|c|}
\hline \multirow[b]{2}{*}{ Condition } & \multirow[b]{2}{*}{$M$} & \multirow[b]{2}{*}{$S D$} & \multicolumn{2}{|c|}{ 95\% Confidence Interval } \\
\hline & & & Lower Bound & Upper Bound \\
\hline \multicolumn{5}{|l|}{ Present } \\
\hline \multicolumn{5}{|l|}{$\mathrm{LVF} / \mathrm{RH}$} \\
\hline Related & 0.56 & 0.02 & 0.52 & 0.61 \\
\hline Unrelated & 0.51 & 0.02 & 0.46 & 0.55 \\
\hline \multicolumn{5}{|l|}{$\mathrm{RVF} / \mathrm{LH}$} \\
\hline Related & 0.59 & 0.02 & 0.54 & 0.64 \\
\hline Unrelated & 0.57 & 0.02 & 0.52 & 0.61 \\
\hline \multicolumn{5}{|l|}{ Absent } \\
\hline \multicolumn{5}{|l|}{$\mathrm{LVF} / \mathrm{RH}$} \\
\hline Related & 0.52 & 0.03 & 0.46 & 0.57 \\
\hline Unrelated & 0.54 & 0.02 & 0.49 & 0.59 \\
\hline \multicolumn{5}{|l|}{$\mathrm{RVF} / \mathrm{LH}$} \\
\hline Related & 0.64 & 0.03 & 0.58 & 0.69 \\
\hline Unrelated & 0.58 & 0.02 & 0.54 & 0.63 \\
\hline
\end{tabular}


Figure 4. Accuracy priming in both visual fields when the placeholder was either absent or present.

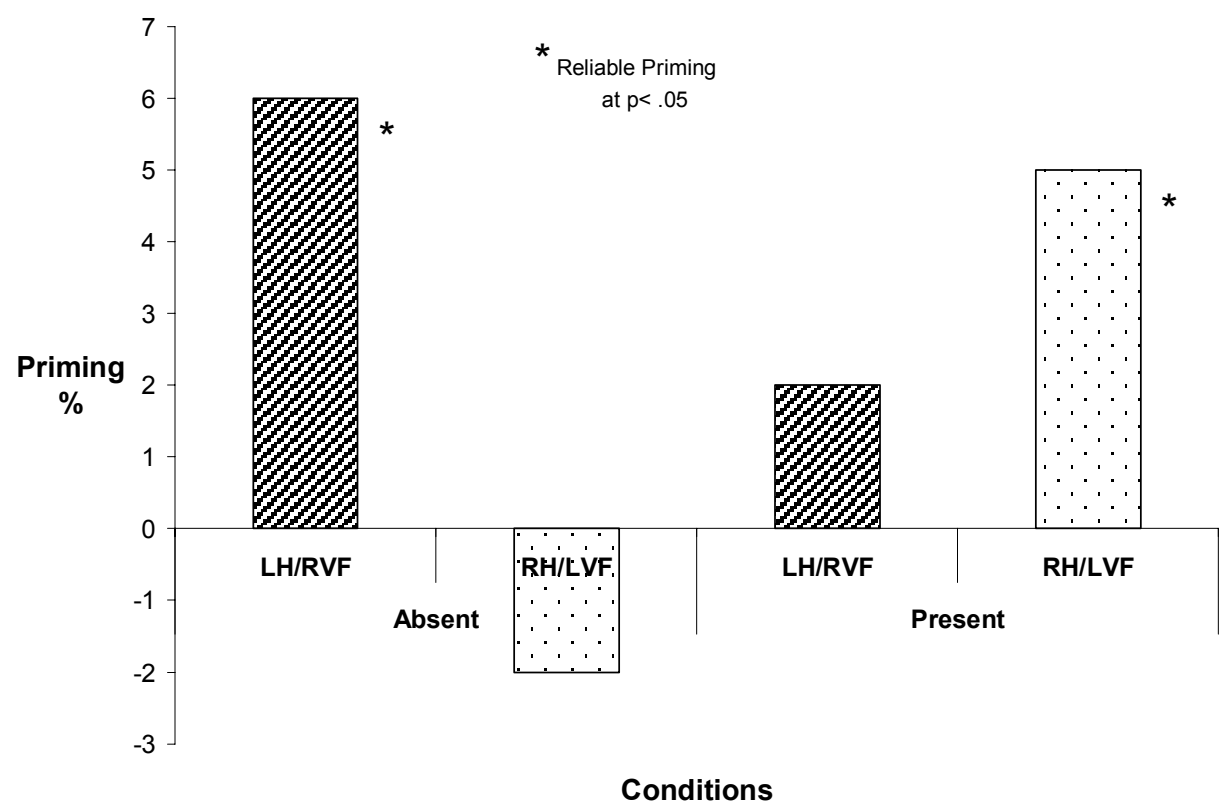

Number of Eye Movements

A Repeated Measures ANOVA was performed on the number of eye movements the participants made per condition. There was a significant main effect for mask, $\mathrm{F}(1,32)=21.65, \mathrm{p}<.01, \mathrm{MS}_{\text {error }}=11.78$; placeholder, $\mathrm{F}(1,32) 15.51, \mathrm{p}$ $<.01, \mathrm{MS}_{\text {error }}=1.91 ;$ and relatedness, $\mathrm{F}(1,32)=17.29, \mathrm{p}<.01, \mathrm{MS}_{\text {error }}=.51$. There was no significant main effect for visual field, $\mathrm{F}(1,32)=.59, \mathrm{p}=.45, \mathrm{MS}_{\text {error }}=1.69$. There was one significant two-way interaction between mask and relatedness, $\mathrm{F}(1,32)$ $=14.63, \mathrm{p}<.01, \mathrm{MS}_{\text {error }}=.30$ (see Table 4). No significant three-way interaction was found.

For the main effect of mask type, participants made more eye movements during the onset mask than the offset mask, Onset: 2.99(.46); Offset: $1.60(.29)$. 
More eye movements occurred when the placeholder was absent than when it was present, Absent: 2.53 (.39); Present: 2.06 (.33). A clearly unexpected finding was that participants made more eye movements when the target was unrelated to the prime than when the target was related to its prime, Unrelated: 2.43 (.37); Related: $2.17(.34)$.

A Neuman-Keuls post-hoc comparison was run on the two-way interaction between mask and relatedness. Reliable differences were found in the onset condition between the unrelated and related conditions. Participants moved their eyes more in the onset condition when the targets were unrelated to the prime than when they were related to the prime. Further post-hoc comparisons were run to ensure any other differences were not missed in the main analysis. No reliable differences were found between left and right visual fields (no laterality differences). There were differences found in the placeholder conditions, however, demonstrating that participants moved their eyes most often when the target was (1) presented using an onset mask, (2) not coupled with a placeholder, and (3) semantically unrelated to the prime (see Figure 5). 


\section{Table 4.}

Table 4

Mask $x$ Relatedness Interaction for Eye Movements per Condition in Experiment 3

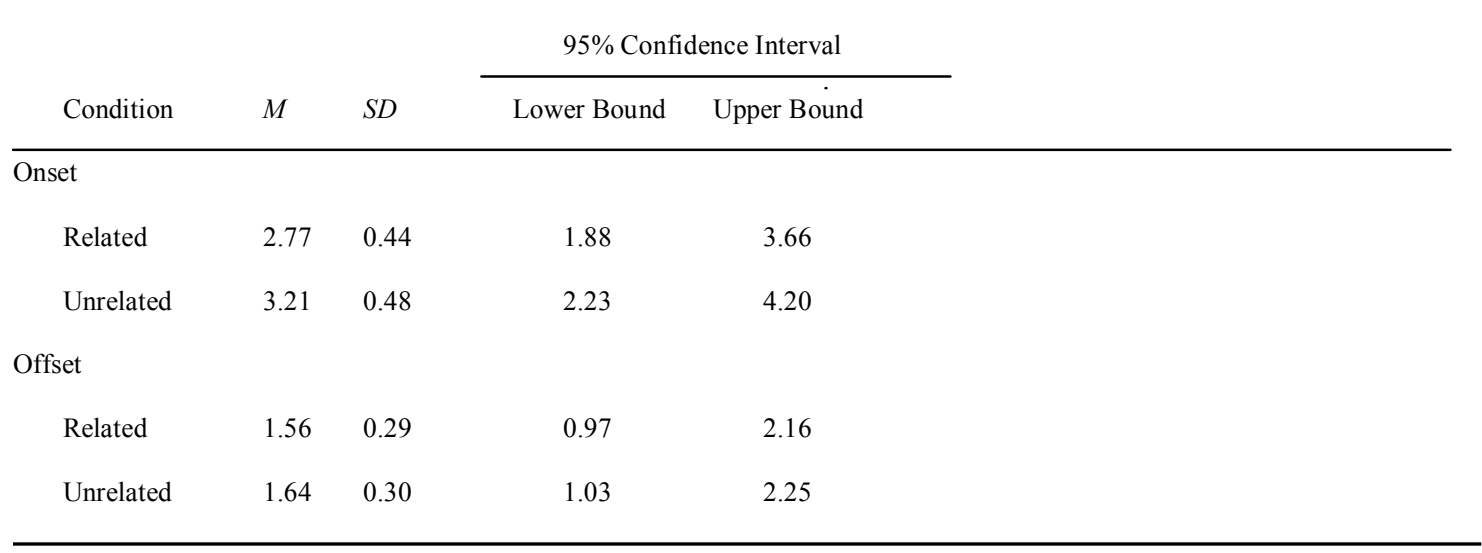

Figure 5. Difference scores for eye movements per condition between unrelated and related trials for each Placeholder and Mask condition in Experiment 3.

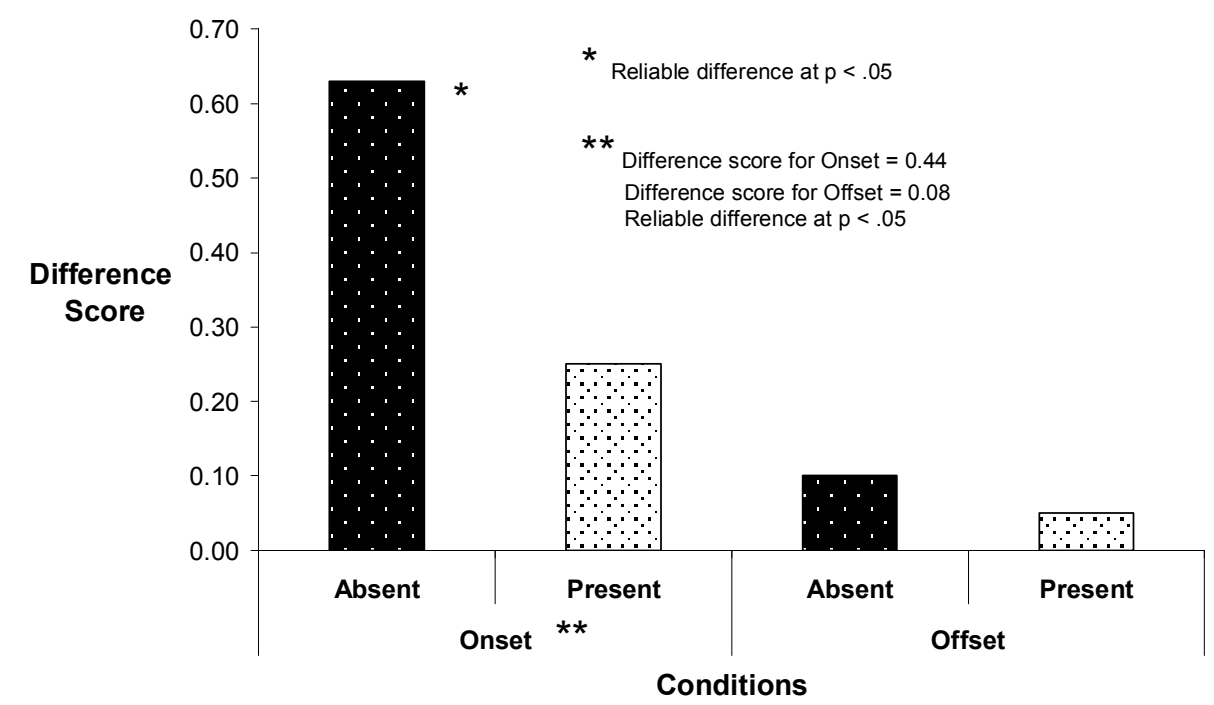




\section{Eye Movement Latency}

A Repeated Measures ANOVA was also performed on the mean latency of onset of the participants' saccades. The only significant main effect occurred for mask type, $\mathrm{F}(1,32)=190.26, \mathrm{p}<.01, \mathrm{MS}_{\text {error }}=926.94$. The participants initiated a saccade earlier during the trials with the onset mask than during trials with the offset mask, Onset: 177.37 (5.53); Offset: 233.49 (4.41). No significant main effects were present for placeholder, $\mathrm{F}(1,32)=.06, \mathrm{p}=.82, \mathrm{MS}_{\text {error }}=331.99$; visual field, $\mathrm{F}(1,32)$ $=.03, \mathrm{p}=.86, \mathrm{MS}_{\text {error }}=294.13$; or relatedness, $\mathrm{F}(1,32)=.03, \mathrm{p}=.86, \mathrm{MS}_{\text {error }}=$ 194.10. There were also no significant interactions found.

\section{Discussion}

For Experiment 3, the most interesting findings arise from the eye movement data. When examining the number of eye movements made per condition the initial goal of the entire study is addressed. Using the dynamic offset procedure developed for Experiment 2, participants' horizontal eye movements were reduced by nearly $50 \%$ from the onset procedure. Indeed, using a more dynamic offset procedure that is more consistent with those typically used in the attention literature (e.g. Atchley, Kramer, \& Hillstrom, 1994; Yantis \& Jonides, 1984) or using a placeholder opposite the target word was effective in reducing eye movements. This finding supports the results from the attention literature which argues that the results from Experiment 1 might have been due to the nature of our offset mask and an unreliable reduction in the onset advantage. In other words, the results from Experiment 3 provide additional evidence that attention is more effectively captured by a new object then by an object 
that emerges from an already previously occupied location. Additionally, post-hoc tests demonstrate that participants move their eyes most often when a target is presented in the traditional setup (onset, no placeholder). This further supports the need to change the traditional DVF paradigm, highlighting how often participants move their eyes and emphasizing that new methods need to be developed in order to prevent the problem in future work. In regards to the latency of the eye movements, the results replicate the findings from Experiment 1, participants initiating an eye movement earlier during the onset procedure than the offset procedure. This difference again highlights the point that the onset and offset procedures could be activating different attentional mechanisms (stimulus-driven vs. goal-driven).

With regards to the behavioral results, participants were more accurate when the onset procedure was used than in the offset procedure, replicating the findings from the first experiment. The left hemisphere advantage and the semantic relatedness main effect, which were not found in Experiment 2, were recovered in the current experiment. The recovery of the language effects is encouraging but must be tempered, however, by the pattern of results for the placeholder. The placeholder did not independently affect accuracy (as was predicted), but it did interact with visual field of presentation and the semantic relatedness of the target. The pattern of results indicate that when the placeholder was absent the left hemisphere showed significant priming and the right hemisphere did not. However, when the placeholder was present the exact opposite occurred - the right hemisphere showed significant priming while the left hemisphere did not. When there is no placeholder, the letter 
string is presented unilaterally (the most typical paradigm) to either the left or right hemisphere. In this scenario, targets are responded to more accurately when presented to the left hemisphere than when presented to the right hemisphere. If a placeholder is present, however, a bilateral presentation occurs with both hemispheres processing letter strings simultaneously. Due to the nature of the lexical decision task, both hemispheres must process a letter string and communicate that information to the other hemisphere before a response decision can be made. In this case, both hemispheres have information about both letter strings and this makes the task more of a global/holistic one rather than a local one because the hemispheres have to quickly process the semantic and lexical properties of both strings and make a decision rather than process those properties of a singular item. This shift from processing at the local level to global processing favors the right hemisphere over the left hemisphere (e.g. Atchley \& Atchley, 1998). Thus, the right hemisphere is able to show semantic priming and higher response accuracies compared to the left hemisphere which is not as adept in this type of scenario.

The final aspect of the results to examine is the continuing theme of low accuracy rates. A detailed accuracy rate breakdown was not conducted here when compared with the previous experiment because using the $50 \%$ cutoff for participants makes no difference in the results (see Experiment 2). Nevertheless accuracy rates for the current study were lower than previously seen, with some of the rates of the experimental conditions overall right at or barely above $50 \%$. With the lower accuracy rates, the behavioral data was used not only for examining the offset mask's 
and placeholder's effect but also as a basic check as to whether or not the language effects lost in Experiment 2 could be recovered.

One unexpected outcome from the study was the influence that the degree of the semantic relationship between the target word and the prime had on participants' eye movements. The data suggest that when the target word is unrelated to the prime, participants are more likely to move their eyes than when the target word is related. Moreover, this effect was strongest when the onset procedure was used and no placeholder was present. This finding argues that the semantic relatedness of the target to the prime affects participants' eye movements most in the traditional DVF setup. Keep in mind that the participants are initiating saccades as soon as $177 \mathrm{~ms}$ (for the onset conditions) and around $230 \mathrm{~ms}$ (for the offset conditions) after the target word is presented. This implies that the semantic relationship between the prime and target may impact very early attention allocation. It is possible that a semantically unrelated target, requiring more time and resources before a response decision can be made (Chiarello, 1998), necessitates allocation of more visual attention resources than a semantically related target. This additional allocation of visual attention might result in initiating an eye movement to the target. While this finding was not replicated with post hoc analysis of the related vs. unrelated conditions in Experiment 1, remember that the first study was methodologically different from Experiment 3 in some key ways. 


\section{General Discussion}

\section{Overview}

Overall, the results from the three experiments paint an interesting picture in regards to both the attention and divided visual field domains. Experiment 1 did not find any difference between the offset or the onset procedures in regards to the number of horizontal eye movements made by the participants. This contradicts the findings from the attention literature, which demonstrated that using offset versus onset masking should effectively reduce the number of eye movements made by an observer (e.g. Boot, Kramer, \& Peterson, 2005; Irwin et al. 2000; Theeuwes et al. 1998, 1999). While this is a concern there were several issues with the mask and procedures that were addressed in Experiment 2, using a new, dynamic offset mask and adding a placeholder in the opposite visual field of the target. Measuring eye movements again in Experiment 3 resulted in data that is more consistent with predicted findings: that the use of a dynamic offset mask causes a significant decrease in horizontal eye movements. Additionally, both Experiment 1 and Experiment 3 showed an eye movement latency difference between the onset and offset masking procedures. This indicates that the two procedures were differentially affecting the initiation of an eye movement. Eye movements inititated during the onset procedure appear to be more rapid and, thus, stimulus-driven, while the offset procedure caused eye movements to be delayed and appear more goal-driven in nature.

Examining the behavioral results, the data from Experiment 1 argued that the basic visual field language findings (both the left hemisphere advantage and 
relatedness effect) can remain intact even when an offset mask is used to present the target words. The accuracy rates for both procedures, however, were lower than expected or desired in DVF studies (what would be a continuing theme). The outcome of the new offset mask and procedure used in Experiment 2 further added to the accuracy rate concerns and created some additional issues. Almost twenty percent of the participants in both tasks (ten in the visual detection task and eight in modified lexical decision task) had accuracies at or below 50\%. When those participants who fell below 50\% were removed in the analysis, accuracy rates were still lower compared to Experiment 1. With or without the low accuracies, both the visual detection task and the modified lexical decision task showed neither a left hemisphere advantage nor a relatedness effect. This raised considerable concern that the new mask was so effective that the task was now too difficult and the attentional manipulations were affecting the nature of the lexical decision task and the language effects. Results from Experiment 3 showed even lower accuracies hovering around 50\%; however, the language effects lost in Experiment 2 were recovered. The disappearance and, subsequent, reappearance of the language effects does raise questions about the reliability of the new paradigm in a DVF language study.

\section{Eye Movements}

The main finding from this study is that horizontal saccades can be effectively reduced in DVF paradigms if a dynamic offset mask is used. Traditional DVF studies have some stimulus flashed on a screen on either visual field while instructing the participants to focus their eyes on a central fixation. When that 
stimulus is presented, two salient properties cause stimulus-driven mechanisms likely capture attention and might cause the observer to move their eyes involuntarily. The first property is that a new object appears in the visual scene when a seemingly blank location suddenly is filled with some new stimuli that pulls an observer's attention away from whatever they were doing and wherever else they were looking (e.g. Jonides \& Yantis, 1988; Yantis, 1993; Yantis \& Egeth, 1994; Yantis \& Hillstrom, 1994; Yantis \& Jonides, 1984, 1990). The second property is that the luminance of that area is altered when that new object appears and causes a reflexive reaction that, again, supersedes what the observer was paying attention to earlier (e.g. Donk \& Theeuwes, 2001, 2003; Godijn \& Theeuwes, 2002; Irwin, Colcombe, Kramer, \& Hahn, 2000; Theeuwes, 1995; Theeuwes, Kramer, Hahn, \& Irwin, 1998; Theeuwes, Kramer, \& Kingstone, 2004). Using an offset mask and a placeholder/filler on the opposite visual field from the target can balance both of those salient features and help to solve the eye movement issues we often observe in DVF research (Gratton, 1998; Jordan, Patching, \& Milner, 1998; Bourne, 2006).

These studies demonstrate that one effective way to reduce horizontal eye movements is to use a dynamic offset mask. The effective offset mask from Experiment 3 was probably more effective because it follows the same principal idea used by Todd and VanGelder (1979) in their no-onset paradigm. In their study, as well as Yantis and Jonides' (1984), a figure eight singleton was the "mask" which then had some combination of vertical and horizontal lines eliminated to create the letters. The current study had the mask as three letter strings, two of which were 
eliminated to reveal the target. While the stimuli in the current study were more complex, the same principle was applied and a similar outcome occurred. This onset advantage over offsets supports the notion that offsets hold less attentional priority than onsets in our visual environment (e.g. Atchley, Kramer, and Hillstrom, 2000; Boot, Kramer, and Peterson, 2005; Donk \& Theeuwes, 2001, 2003; Jonides \& Yantis, 1988; Theeuwes, Kramer, Hahn, and Irwin (1998); Theeuwes, Kramer, Hahn, Irwin, and Zelinksy (1999); Yantis, 1993; Yantis \& Egeth, 1994; Yantis \& Hillstrom, 1994; Yantis \& Jonides, 1984, 1990). Not only did the data support it with the eye movements (reducing the number of movements by almost one half) but also with the behavioral findings (onset targets were responded to more accurately than offset targets in both Experiment 1 and Experiment 3).

The other aspect of the procedure that proved effective was the use of the placeholder or filler in the opposite visual field from the target letter string. By having a non-word letter string that was matched for length, covered and revealed the same way as the target string, the luminance change on either side of the central fixation was perceived to be equal. With the luminance change in the two visual fields perceived as similar, the participants seemed to have no salient properties available for cuing an attentional shift and, therefore, were less likely to move their eyes toward a specified string (e.g. Theeuwes, 1995). When no placeholder was present, however, the luminance changed drastically on one side of the visual field, leading participants to make a saccade more often. 
While the mask and placeholder procedures had an effect on the number of eye movements in Experiment 3, the common finding in both Experiments 1 and 3 was the latency difference in initiating a saccade in the onset versus the offset conditions. When a simple onset (whether using a placeholder or not) target was presented, participants initiated a saccade about $175-185 \mathrm{~ms}$ after the target onset in an attempt (presumably) to process it. When a target was offset from the mask, however, participants initiated a saccade roughly $210-235 \mathrm{~ms}$ after the target appeared. This leads to speculation that the two procedures might be reflecting different attentional mechanisms. Past research suggests that reflexive saccades occur approximately between 180 and 200ms after stimuli is presented (Becker, 1991). That timeframe not only matches with that of the saccades in the onset conditions but also further supports that onsets activate stimulus-driven mechanisms that involuntarily capture an observer's attention. Goal-oriented or programmed saccades occur after about 200ms (Becker, 1991), which might fit with the saccade latencies found during the offset condition. Once the target and the placeholder are presented the observer would take between $140-200 \mathrm{~ms}$ to decide which letter string is the target and then program a saccade to the corresponding visual field. Noting the low accuracy rates, however, that programmed saccade would not help them as the stimuli are only up there for $185 \mathrm{~ms}$. Nevertheless, if this distinction is indeed demonstrated through the different procedures, the onsets would cause reflexive saccades, which are operated via the superior colliculus (Reingold \& Stampe, 2002; e.g. Munoz \& Wurtz, 1992) and the offsets might cause programmed saccades via top-down 
systems such as the frontal eye fields (Reingold \& Stampe, 2002; e.g. Segraves \& Goldberg, 1987).

\section{Semantic Relatedness Main Effect in Experiment 3}

As discussed in Experiment 3, the offset masking procedure had predicted effects on the number of saccades made by the participants, however, an unexpected effect also occurred in that the semantic relatedness of the lateralized target to the central prime was also related to a change in the number of eye movements. The data suggest that when the target word was semantically unrelated to the target, participants were more likely to initiate a saccade than when the target word was semantically related. Additionally, this effect was strongest when the targets were presented using the traditional (onset procedure without an opposite visual field placeholder) DVF paradigm. Post hoc analysis of the same variable in Experiment 1 did not replicate that finding, however that result is tempered by several key differences between Experiments 1 and 3.

The first difference is that the nature of the offset mask in Experiment 1 was of a very different construction as the mask used in Experiment 3. The mask used in the latter experiment was dynamic and a closer approximation of the offsets used in the past to demonstrate the onset advantage, while the first experiment used a mask that was not as complex (letter boxes instead of actual letter strings to "obscure" the targets) and which was held constant in appearance across the experiment. In addition to using true offsets, using placeholders on half of the trials in Experiment 3 created vastly different conditions and a significantly altered modified lexical 
decision task that the participants completed, as compared to the traditional lexical decision task that the participants completed in Experiment 1. The other difference was a change in the presentation durations for the primes and targets in Experiment 3, which allowed for better comparisons of the behavioral data from past DVF studies (Chiarello, 1988).

Noting these differences, there are several possibilities that stem from the semantic relatedness findings in Experiment 3. The first possibility is a theoretical one, with the current evidence suggesting that mechanisms mediating lexical and semantic access directly interact with the mechanisms mediating allocation of visual spatial attention. This interaction then allows visual attention mechanisms to mediate eye movements to the stimuli. The data make an even stronger case for this when examining the mask and relatedness interaction. In the offset masking condition, eye movements are not affected by the semantic relatedness of the targets because that procedure disrupts the visual attention system's ability to focus on the target. Furthermore, the post hoc comparisons showed that the relatedness effect is strongest in the onset masking condition when no placeholder is present. Again, the placeholder disrupts the visual attention system's ability to focus on the target word but when it is absent the attention system is not hampered and can more easily process (in this case the semantic information of) the target word. Taking all of this into account, when the targets are presented in the traditional DVF paradigm the visual attention system can fully interact with lexical and semantic access (no disruption of the visual attention system occurs). After being presented with the 
prime, if a semantically related target appears it has already been primed so processing it and making an appropriate response should be fairly quick and should require few resources. If the target is semantically unrelated, however, it should not be primed. The lack of priming means processing time of the word would be longer and require more resources in order to complete the task in the time required for the task (Chiarello, 1998). If more resources are required to process the semantically unrelated word, it would be beneficial for the visual attention system to allocate more resources to allow for lexical and semantic access to occur within the constraints of the task. A way to allocate more attention to the target is to initiate an eye movement so that the target can be seen and processed more effectively in the central/foveal vision rather than in the lateral/peripheral vision. This idea is supported by the work of Sereno, Brewer, \& O’Donnell (as cited in Dell'Acqua, Pesciarelli, Jolicœur, Eimer, $\&$ Peressotti, 2007) on reading. They found that fixation times were longer for words that were semantically incongruent in the context of the passage being read compared with words that were semantically congruent. Again, more allocation of visual attention is needed to process the semantically unrelated (incongruent in the case of the aforementioned study) and that can be accomplished by moving one's eyes and centrally fixating on the critical stimuli. While the interaction mentioned above is an interesting proposition, the more remarkable issue to keep in mind is that the data in the study suggest this interaction between lexical/semantic access, visual attention and eye movements is taking place (on average) in a little under $180 \mathrm{~ms}$. 
Despite the suggested time course of the interaction between visual attention and lexical and semantic access seeming remarkable, recent evidence adds some support. An ERP study conducted by Dell'Acqua and colleagues (2007) examined the modulation of the N2pc component by semantically related or unrelated word pairs. They presented 120 equal length Italian word pairs (simultaneously and bilaterally) for only $85 \mathrm{~ms}$. One word was designated the target and the other the distractor, each presented in a different color (e.g. red and green), with participants being instructed on which color word to attend to. Results showed that the N2pc (emerging between 170-180ms) was more negative on trials in which the word pairs were semantically unrelated than when they were related. Furthermore, the N2pc component was largest over occipito-parietal regions (at P7 and P8 channel sites) and it is thought to originate from the extrastriate visual cortex. The authors conclude that a rapid semantic analysis of the target is sent to the visual areas and then biases the allocation of visual attention. Their conclusion provides strong evidence to the existence of this interaction between visual attention and lexical/semantic access and even implies that semantic information can modulate (systematically) the distribution of that attention. While the converging evidence of the current study and the work of Dell'Acqua and colleagues create an interesting argument more studies need to be conducted to refine the time-course and parse out the specifics of the nature of the relationship between semantic access and visual attention.

Along with this theoretical possibility comes the implication that aspects of language could be more thoroughly explored measuring eye movements in DVF 
studies. Numerous studies in the past have used eye trackers in language studies. Some work has focused on saccades effect on language and other cognitive processes (e.g. Irwin 1998; Irwin \& Carlson-Radvansky, 1996; Irwin, Carlson-Radvansky, \& Andrews, 1995; Rayner, McConkie, \& Ehrlich, 1978; Rayner, McConkie, \& Zola, 1980). Most of the work using eye movements in language has been with discourse reading, examining variables such as fixation/gaze duration, scanning time (for a line or several words instead of one word), and the amount of rereading that occurs. While that line of research is very beneficial, it cannot be done in a DVF paradigm (in terms of presenting a full-length sentence; laterality can only be examined in sentences by having a single word from the sentence, typically the final word, presented laterally). Up to this point DVF researchers interested in language are limited to behavioral and/or brain imaging techniques, but the addition of eye movement data to examine lexical access and semantics could bring yet another tool to the study of lateralization.

\section{Accuracy Rates}

The issue throughout the study that raises the largest concern is the low accuracy rates shown by participants, which are a problem for any researcher (unless they have a priori reasons for creating tasks that result in chance performance). Unfortunately, the low accuracies (1) make the data less interpretable, (2) did not allow for analysis (even measuring in Experiments 2 and 3) of the response time data, and (3) perhaps demonstrate that the specific conditions of the task are simply too difficult for the participants. 
When looking at the accuracies from the three separate experiments, Experiment 1 had the highest rates with the onset trials being detected at nearly $70 \%$ compared with offset trials at $63 \%$ accuracy. These rates are below the usual accepted rates of $75 \%$ and above (e.g. Chiarello, Kacinik, Otto, Manowitz, \& Leonard, 2004), which is made more troubling given that the presentation times for the primes and targets were longer than usual. The accuracy rates in Experiment 2 and Experiment 3 were even lower, hovering close to the 50\% accuracy rate that we would expect if the subject was responding randomly to the task. This suggests that the new mask procedures made the tasks so difficult that the participants might simply have been guessing on many of the trials and only were able to effectively decide on about half the trials. This difficulty would hurt performance further by causing continuing discouragement when the feedback indicated the answer was incorrect. Becoming more discouraged as the session continued seems to be a similar situation to learned helplessness, which might have evolved to the point that even the easiest trials were perceived as difficult.

There appear to be two main reasons why the new paradigm made the tasks tougher for the participants. The first reason is the nature of the modified lexical decision task. As discussed earlier, by placing a different letter string in each visual field we are asking participants to make a lexical decision regarding both letter strings simultaneously. This morphs the task from a simple lexical decision into a type of word detection task that involves each hemisphere having to lexically and, possibly, semantically (if the letter string is a word) process the letter strings. In this way, the 
task is testing interhemispheric communication and bilateral language processing instead of the intended unilateral approach, which conflicts with the very idea of examining laterality in language.

The second reason for the difficulty stems from the direct manipulation of the new mask and procedures and its effects on attentional mechanisms. By using offsets and placeholders as a way to reduce attentional capture, the stimuli would be perceived as more difficult to see. While a letter string that is offset from a mask has identical physical properties to that of a new letter string that is onset in the visual scene, the offset letter string will not capture our attention as effectively. When our attention is not captured by a specific object, it makes processing that object much more difficult. This difficulty in attending and processing the object can give us a less assured perception and feeling that we can actually see the object. As a result, performance becomes poor.

While the overall accuracy rates are low in order to better compare the results from this study to those of earlier DVF work, we should more closely examine the specific condition from Experiment 3 in which participants responded to onset targets that were not accompanied by an opposite visual field placeholder. This specific condition most resembles past DVF language paradigms so these data might give us some indication of whether or not the results we are seeing are only due to our experimental manipulations or if, alternatively, we are also examining a group of participants that are less accurate overall. Analyzing the results show that even in the onset condition without the placeholder, accuracy rates are still below expected. The 
trials presented in the $\mathrm{LVF} / \mathrm{RH}$ were responded to accurately $55 \%$ of the time, while $\mathrm{RVF} / \mathrm{LH}$ trials were detected correctly $63 \%$. Thus, these results could be an indication of an overall lack of attention or motivation in our participants. However, this does not provide a fully satisfying explanation given that we have replicated this poor level of performance in three independent samples of participants. The most parsimonious explanation seems to be that in this experimental context where (1) the lexical decision task is clearly different and more difficult and (2) the stimuli are harder to see, participants do poorly. To test this hypothesis in the future we could use a fully blocked design.

\section{Implications for DVF Methodology}

With all of the concerns and theoretical implications, the practical and methodological outcome from this study is that using offset masks and placeholders does reduce horizontal eye movements made by participants in a DVF task. If a researcher uses a DVF paradigm, especially if measuring ERPs, this new masking procedure and the issues it raises should be considered. The most interesting issue discussed (in the section titled "Semantic Relatedness Main Effect in Experiment 3") might be the possible interaction between the visual attention system and lexical/semantic access. While more work is needed to confirm and/or refine those findings, it highlights new issues that needed to be considered by researchers in the DVF domain. Additionally, due to the concerns raised in the section titled "Accuracy Rates," the current offset paradigm needs further examination before it can be a practical recommendation for DVF work in language processing. The language 
results obtained using the offset mask and placeholder methods are currently not robust enough to be a good foundation for laterality research. Couple this with the low accuracy rates and the subsequent inability to use reaction times and any researcher in the domain would be weary to currently incorporate the new paradigm with a current one. However, issues such as the stimulus-driven mechanisms (both attentional and physiological) behind horizontal saccades and attentional capture should be better understood and incorporated in the interpretation of outcomes in the DVF domain in the future.

The main focus of the current study was the elimination of horizontal saccades which are a well documented problem in DVF research, especially when concurrently measuring ERPs. The offset paradigm used in the current study is a very effective method for correcting for horizontal eye movements that does not require any (1) online observation by the researcher, (2) corrective mathematical models applied after the data is recorded, or (3) the use of additional equipment (eye trackers). Overall, we conclude that while the new paradigm from this study is effective the concerns and questions raised are still too numerous for it to be a complete solution to the problem.

\section{Future Directions}

The questions generated by the current research leads to at least two lines of future research. The first is aimed at a better understanding of the semantic relatedness effect on eye movements observed in Experiment 3. Before any broad theoretical conclusions can be gleaned from the results, the finding must be 
replicated. If the finding is, indeed, robust then a long line of research could stem from this study and drastically impact several domains. Theories on semantic and lexical access and visual attention might be refined to account for a possible interaction occurring in a previously undocumented time-course. In addition, measuring eye movements could become more integral in defining and understanding a wider array of language processes (e.g. semantics, syntax, and morphology instead of just examining discourse reading).

Another set of future studies should examine the relationship between attentional mechanisms and language processes using the DVF paradigm. The offset paradigm used here had a large impact on the typical language effects in DVF work, so the question is why. Is the new task and paradigm creating a more atypical bilateral and divided attention/dual task versus the typical unilateral and single tasks used, as discussed earlier? Areas to consider should include attentional bias and lateralization of attention and the roles each play in the typical DVF language work. 


\section{References}

Atchley, P., Kramer, A.F., \& Hillstrom, A.P. (2000). Contingent capture for onsets and offsets: attentional set for perceptual transients. Journal of Experimental Psychology: Human Perception \& Performance, 26, 594-606.

Atchley, R.A. \& Atchley, P. (1998). Hemispheric specialization in the detection of subjective objects. Neuropsychologia, 36, 1373-1386.

Atchley, R.A., Burgess, C., \& Keeney, M. (1999). The effect of time course and context on the facilitation of semantic features in the cerebral hemispheres. Neuropsychology, 13, 1-15.

Becker, W. (1989). Metrics. In R.H. Wurtz \& M.E. Goldberg (Eds.), The neurobiology of saccadic eye movements (pp. 13-67). Amsterdam: Elsevier.

Beeman, M., \& Chiarello, C. (Eds.). (1998). Right Hemisphere Language Comprehension: Perspectives from Cognitive Neuroscience. Mahwah: Lawrence Erlbaum Associates, Inc.

Boot, W.R., Kramer, A.F., \& Peterson, M.S. (2005). Oculomotor consequences of abrupt object onsets and offsets: Onsets dominate oculomotor capture. Perception \& Psychophysics, 67, 910-928.

Bourne, V.J. (2006). The divided visual field paradigm: Methodological considerations. Laterality, 11, 373-393.

Chiarello, C. (1998). Semantic priming in the intact brain: Separate roles for the right and left hemispheres? In C. Chiarello (Ed.), Right hemisphere contributions to lexical semantics. Berlin, Germany: Springer-Verlag. 
Chiarello, C., Kacinik, N., Manowitz, B., Otto, R., \& Leonard, C. (2004). Cerebral asymmetries for language: Evidence for structural-behavioral correlations. Neuropsychology, 18, 219-231.

Cole, G.G., Kentridge, R.W., Gellatly, A.R.H., \& Heywood, C.A. (2003).

Detectability of onsets versus offsets in the change detection paradigm. Journal of Vision, 3, 22-31.

Dell'Acqua, R., Pesciarelli, F., Jolicœur, P., Eimer, M., \& Peressotti, F. (2007). The interdependence of spatial attention and lexical access as revealed by early asymmetries in occipito-parietal ERP activity. Psychophysiology, 44, 436443.

Donk, M., \& Theeuwes, J. (2001). Visual marking beside the mark: Prioritizing selection by abrupt onsets. Perception \& Psychophysics, 63, 891-900.

Donk, M., \& Theeuwes, J. (2003). Prioritizing selection of new elements: Bottom-up versus top-down control. Perception \& Psychophysics, 65, 1231-1242.

Enns, J.T., Austen, E.L., Di Lollo, V., Rauschenberger, R., \& Yantis, S. (2001). New objects dominate luminance transients in setting attentional priority. Journal of Experimental Psychology: Human Perception \& Performance, 27, 12871302.

Folk, C.L., Remington, R.W., \& Johnston, J.C. (1992). Involuntary covert orienting is contingent on attentional control settings. Journal of Experimental Psychology: Human Perception \& Performance, 18, 1030-1044. 
Folk, C.L., Remington, R.W., \& Wright, J.H. (1994). The structure of attentional control: Contingent attentional capture by apparent motion, abrupt onset, and color. Journal of Experimental Psychology: Human Perception \& Performance, 20, 317-329.

Godijn, R., \& Theeuwes, J. (2002). Programming of endogenous and exogenous saccades: Evidence for a competitive integration model. Journal of Experimental Psychology: Human Perception \& Performance, 28, 1039-1054.

Gratton, G. (1998). Dealing with artifacts: The EOG contamination of the eventrelated brain potential. Behavior Research Methods, Instruments, \& Computers, 30, 44-53.

Gratton, G., Coles, M.G.H, \& Donchin, E. (1983). A new method for off-line removal of ocular artifact. Electroencephalography and clinical Neurophysiology, 55, 468-484.

Hickey, C., McDonald, J.J., \& Theeuwes, J. (2006). Electrophysiological evidence of the capture of visual attention. Journal of Cognitive Neuroscience, 18, 604613.

Hoffman, J.E., \& Subramanium, B. (1995). The role of visual attention in saccadic eye movements. Perception \& Psychophysics, 57, 787-795.

Humphreys, G.W., Olivers, C.N.L., \& Yoon, E.Y. (2006). An onset advantage without a preview benefit: Neuropsychological evidence separating onset and preview effects in search. Journal of Cognitive Neuroscience, 18, 110-120. 
Irwin, D.E. (1998). Lexical processing during saccadic eye movements. Cognitive Psychology, 36, 1-27.

Irwin, D.E., \& Carlson-Radvansky, L.A. (1996). Cognitive suppression during saccadic eye movements. Psychological Science, 7, 88.

Irwin, D.E., Carlson-Radvansky, L.A., \& Andrews, R.V. (1995). Information processing during saccadic eye movements. Acta Psychologica,90, 261-273.

Irwin, D.E., Colcombe, A.M., Kramer, A.F., Hahn, S. (2000). Attentional and oculomotor capture by onset, luminance, and color singletons. Vision Research, 40, 1443-1458.

Jonides, J., \& Yantis, S. (1988). Uniqueness of abrupt onset in capturing attention. Perception \& Psychophysics, 43, 346-354.

Jordan, T.R., Patching, G.R., \& Milner, A.D. (1998). Central fixations are inadequately controlled by instructions alone: Implications for studying cerebral asymmetry. The Quarterly Journal of Experimental Psychology, 51A, 371-391.

Kutas, M., \& Hillyard, S.A. (1980). Reading senseless sentences: Brain potentials reflect semantic incongruity. Science, 207, 203-205.

Luck, S. J., Girelli, M., McDermott, M. T., \& Ford, M. A. (1997). Bridging the gap between monkey neurophysiology and human perception: An ambiguity resolution theory of visual selective attention. Cognitive Psychology, 33,6487. 
Luck, S. J., \& Hillyard, S. A. (1994). Spatial filtering during visual search: Evidence from human electrophysiology. Journal of Experimental Psychology: Human Perception \& Performance, 20, 1000-1014.

McConkie, G., Reddix, M. \& Zola, D. (1992). Perception and cognition in reading: Where is the meeting point? In K. Rayner (Ed.), Eye movements and visual cognition: Scene perception and reading (pp. 293-303). New York: SpringerVerlag.

McConkie, G., Underwood, N., Zola, D., \& Wolverto, G. (1985). Some temporal characteristics of processing during reading. Journal of Experimental Psychology: Human Perception \& Performance, 11, 168-186.

Munoz, D.P., \& Wurtz, R.H. (1992). Role of the rostral superior colliculus in active visual fixation and execution of express saccades. Journal of Neurophysiology, 67, 1000-1002.

Patching, G.R., \& Jordan, T.R. (1998). Increasing benefits of eye-tracking devices in divided visual field studies of cerebral asymmetry. Behavior Research Methods, Instruments, \& Computers, 30, 643-650.

Rayner, K., McConkie, G., \& Ehrlich, S. (1978). Eye movements and integrating information across fixations. Journal of Experimental Psychology: Human Perception \& Performance, 4, 529-544.

Rayner, K., McConkie, G., \& Zola, D. (1980). Integrating information across eye movements. Cognitive Psychology, 12, 206-226. 
Reingold, E.M., \& Stampe, D.M. (2002). Saccadic inhibition in voluntary and reflexive saccades. Journal of Cognitive Neuroscience, 14, 371-388.

Samuel, A.G., \& Weiner, S.K. (2001). Attentional consequences of object appearance and disappearance. Journal of Experimental Psychology: Human Perception \& Performance, 27, 1433-1451.

Segraves, M.A., \& Goldberg, M.E. (1987). Functional properties of corticotectal neurons in the monkey's frontal eye field. Journal of Neurophysiology, 58, 1387-1419.

Sereno, S. C., Brewer, C. C., \& O’Donnell, P. J. (2003). Context effects in word recognition: Evidence for early interactive processing. Psychological Science, 14, 328-333.

Steinmann, B.A., Steinmann, S.B., \& Lehmkuhle, S. (1997). Transient visual attention is dominated by the magnocellular stream. Vision Research, 37, 1723.

Theeuwes, J. (1991). Cross-dimensional perceptual selectivity. Perception \& Psychophysics, 50, 184-193.

Theeuwes, J. (1991). Exogenous and endogenous control of attention: The effect of visual onsets and offsets. Perception \& Psychophysics, 49, 83-90.

Theeuwes, J. (1995). Abrupt luminance change pops out; abrupt color change does not. Perception \& Psychophysics, 57, 637-644. 
Theeuwes, J., Kramer, A.F., Hahn, S. \& Irwin, D.E. (1998). Our eyes do not always go where we want them to go: Capture of the eyes by new objects. Psychological Science, 9, 379-385.

Theeuwes, J., Kramer, A.F., Hahn, S., Irwin, D.E., \& Zelinksy, G.J. (1999). Influence of attentional capture on oculomotor control. Journal of Experimental Psychology: Human Perception \& Performance, 25, 1595-1608.

Theeuwes, J., Kramer, A.F., \& Kingstone, A. (2004). Attentional capture modulates perceptual sensitivity. Psychonomic Bulletin \& Review, 11, 551-554.

Todd, J.T., \& Van Gelder, P. (1979). Implications of a transient-sustained dichotomy for the measurement of human performance. Journal of Experimental Psychology: Human Perception \& Performance, 5, 625-638.

Watson, D.G., \& Humphreys, G.W. (1997). Visual marking: Prioritizing selection for new objects by top-down attentional inhibition of old objects. Psychological Review, 104, 90-122.

Van Zoest, W., Donk, M., \& Theeuwes, J. (2004). The role of stimlus-driven and goal-driven control in saccadic visual selection. Journal of Experimental Psychology: Human Perception \& Performance, 30, 746-759.

Yantis, S. (1993). Stimulus-driven attentional capture. Current Directions in Psychological Science, 2, 156-161.

Yantis, S., \& Egeth, H.E. (1994). Visual salience and stimulus-driven attentional capture. Investigative Opthalmology \& Visual Science, 35, 1619. 
Yantis, S., \& Hillstrom, A.P. (1994). Stimulus-driven attentional capture: Evidence from equiluminant visual objects. Journal of Experimental Psychology: Human Perception \& Performance, 20, 95-107.

Yantis, S., \& Jonides, J. (1984). Abrupt visual onsets and selective attention: Evidence from selective search. Journal of Experimental Psychology: Human Perception \& Performance, 10, 601-621.

Yantis, S., \& Jonides, J. (1990). Abrupt visual onsets and selective attention: Voluntary versus automatic allocation. Journal of Experimental Psychology: Human Perception \& Performance, 16, 121-134.

Yantis, S., \& Jonides, J. (1996). Attentional capture by abrupt onsets: New perceptual objects or visual masking? Journal of Experimental Psychology: Human Perception \& Performance, 22, 1505-1513. 